ARTICLE

https://doi.org/10.1038/s41467-019-09908-1

\title{
Regeneration of severely damaged lungs using an interventional cross-circulation platform
}

\author{
Brandon A. Guenthart ${ }^{1,2,9}$, John D. O'Neill, ${ }^{1,9}$ Jinho Kim¹,3, Dawn Queen', Scott Chicotka², Kenmond Fung ${ }^{4}$, \\ Michael Simpson², Rachel Donocoff ${ }^{5}$, Michael Salna², Charles C. Marboe ${ }^{6}$, Katherine Cunningham', \\ Susan P. Halligan', Holly M. Wobma', Ahmed E. Hozain ${ }^{1,2}$, Alexander Romanov ${ }^{5}$, \\ Gordana Vunjak-Novakovic (i] ${ }^{1,7} \&$ Matthew Bacchetta ${ }^{1,8}$
}

The number of available donor organs limits lung transplantation, the only lifesaving therapy for the increasing population of patients with end-stage lung disease. A prevalent etiology of injury that renders lungs unacceptable for transplantation is gastric aspiration, a deleterious insult to the pulmonary epithelium. Currently, severely damaged donor lungs cannot be salvaged with existing devices or methods. Here we report the regeneration of severely damaged lungs repaired to meet transplantation criteria by utilizing an interventional crosscirculation platform in a clinically relevant swine model of gastric aspiration injury. Enabled by cross-circulation with a living swine, prolonged extracorporeal support of damaged lungs results in significant improvements in lung function, cellular regeneration, and the development of diagnostic tools for non-invasive organ evaluation and repair. We therefore propose that the use of an interventional cross-circulation platform could enable recovery of otherwise unsalvageable lungs and thus expand the donor organ pool.

\footnotetext{
${ }^{1}$ Department of Biomedical Engineering, Columbia University Medical Center, Columbia University, New York, NY 10032, USA. ${ }^{2}$ Department of Surgery, Columbia University Medical Center, Columbia University, New York, NY 10032, USA. ${ }^{3}$ Department of Biomedical Engineering, Stevens Institute of Technology, Hoboken, NJ 07030, USA. ${ }^{4}$ Department of Clinical Perfusion, Columbia University Medical Center, Columbia University, New York, NY 1003, USA. ${ }^{5}$ Institute of Comparative Medicine, Columbia University Medical Center, Columbia University, New York, NY 10032, USA. ${ }^{6}$ Department of Pathology and Cell Biology, Columbia University Medical Center, Columbia University, New York, NY 10032, USA. 7 Department of Medicine, Columbia University Medical Center, Columbia University, New York, NY 10032, USA. ${ }^{8}$ Department of Thoracic and Cardiovascular Surgery, Vanderbilt University, Nashville, TN 37232, USA. ${ }^{9}$ These authors contributed equally: Brandon A. Guenthart, John D. O'Neill. Correspondence and requests for materials should be addressed to G.V-N. (email: gv2131@cvolumbia.edu) or to M.B. (email: matthew.bacchetta@vumc.org)
} 
O rgan transplantation has overcome significant technical, immunological, and ethical hurdles to become a lifesaving treatment ${ }^{1}$. The full potential of organ transplantation remains constrained by the global shortage of organsa problem that has been described as "the greatest crisis facing biomedicine today" 2 . Notably, up to $80 \%$ of donated lungs are not utilized $^{3}$, often as a result of injury at the time of death (e.g., trauma, pulmonary contusion, aspiration, ventilator-associated lung injury). Major efforts to expand the donor pool are underway $^{4-6}$, including ex vivo lung perfusion (EVLP), which is under clinical investigation as a means to evaluate and improve marginal quality donor lungs ${ }^{7}$. With the use of hyperosmotic and blood-based perfusates, EVLP has been shown to decrease pulmonary edema and enable $\sim 6 \mathrm{~h}$ of normothermic perfusion prior to transplantation ${ }^{8,9}$. EVLP is also used as an investigational platform for the delivery of therapeutic agents including antibiotics $^{10}$, pulmonary surfactant ${ }^{11,12}$, immunomodulatory viral vectors $^{13,14}$, and mesenchymal stem cells ${ }^{15,16}$.

Despite the clinical use of EVLP since its introduction in 2012 and expansion of criteria for donor lungs, the annual number of lung transplants in the United States has not dramatically increased ${ }^{17}$. Consequently, there is a compelling need to extend organ recovery capabilities beyond the limited category of marginal lungs to severely damaged lungs, such as those injured by aspiration of gastric contents (hydrochloric acid, bile acids, gastric enzymes, and food particulates ${ }^{18,19}$ ). Gastric aspiration results in damage to the pulmonary epithelium, leading to pneumonitis and respiratory insufficiency ${ }^{18}$. Previous attempts to recover lungs injured by gastric aspiration using EVLP have been unsuccessful $^{20}$, having resulted in increased edema ${ }^{21}$ and failure to reduce levels of inflammatory cytokines ${ }^{22}$ or improve tissue morphology $^{23}$ (Supplementary Table 1-3).

We previously reported the development of an extracorporeal organ support platform that utilized cross-circulation to radically extend the duration of time that lungs can be maintained outside the body (normothermic perfusion time $37.7 \pm 1.8 \mathrm{~h}$; total extracorporeal preservation time $56.2 \pm 0.1 \mathrm{~h})^{24}$. Following these prolonged extracorporeal preservation times, lungs met all transplantation criteria and showed maintenance of pulmonary vasoregulation, microvascular and bronchial epithelial tight junctions, alveolar type II pneumocyte surfactant recycling, airway submucosal gland secretory activity, and a coordinated mucociliary escalator. Based on these results, we hypothesized that normothermic perfusion via crosscirculation could enable therapeutic interventions leading to functional recovery and regeneration of severely damaged lungs.

In this study, we use an established clinically relevant large animal model of gastric aspiration to recapitulate severe injury that currently renders a large number of donated lungs unsalvageable for clinical transplantation. We investigate the ability of the cross-circulation platform to: (i) provide prolonged normothermic extracorporeal support of severely damaged lungs outside the body, and (ii) enable continuous assessment of lung regeneration at the molecular, cellular, and tissue levels. Severely damaged lungs are subjected to multiple therapeutic interventions -bronchoalveolar lavage, surfactant replacement, and alveolar recruitment-on an interventional cross-circulation platform that provided physiologic systemic regulation. We also apply noninvasive diagnostics (exosomes, surface thermography) in extracorporeal lungs on cross-circulation support, and establish benchmarks of lung repair and regeneration and corresponding scope of analyses (Supplementary Table 4).

\section{Results}

Experimental design. To induce lung injury, gastric contents $\left(2 \mathrm{~mL} \mathrm{~kg}^{-1} ; \mathrm{pH} 2\right)$ were delivered into a single lung of an anesthetized swine via flexible video bronchoscopy, and an acute injury was allowed to develop for $6 \mathrm{~h}$ (Fig. 1a; Supplementary Movie 2). Progression of lung injury was assessed every $2 \mathrm{~h}$ by radiography, bronchoscopy, bronchoalveolar lavage fluid analysis, and serologic and hemogas analyses (Fig. 2, Supplementary Table 5). Upon explant, extensive lung consolidation, cellular derangement, peribronchial edema, and regions of alveolar hemorrhage, edema, and pervasive infiltration of leukocytes were observed (Supplementary Fig. 1). Lungs were cannulated, and pulmonary venous drainage was managed with cannulas placed through a vascular bio-bridge to enable single lung functional assessment (i.e., gas exchange in injured and control lungs; Fig. 1c-g; Supplementary Fig. 2a-h). An endotracheal tube was inserted and secured in the extracorporeal lungs to enable continuous ventilation throughout the duration of the study. Periodic use of bronchial blockers enabled single lung assessment of compliance of injured and control lungs (Fig. 1h-j; Supplementary Fig. $2 \mathrm{i}-\mathrm{m}$ ). Following cannulation of the internal jugular veins of the recipient, lungs were connected to the extracorporeal circuit and cross-circulation was established. All cross-circulation procedures were conducted in an operating room and utilized clinical equipment and supporting devices (Supplementary Fig. 3).

Validation of in vivo lung injury after gastric aspiration. Video bronchoscopy was used to confirm unilateral delivery of gastric contents into lungs (Fig. 2b; Supplementary Movie 2). Six hours after in vivo delivery of gastric contents, chest X-rays showed marked unilateral lung injury characterized by increased radiopacity, basilar atelectasis, and areas of consolidation with air bronchograms (Fig. 2a). Bronchoscopy revealed severe unilateral airway inflammation, edema, and mucus secretions (Fig. 2b) resulting from the insult of gastric contents. Lung donors experienced hemodynamic instability (increased heart rate, decreased MAP; Fig. 2c, d), significantly decreased gas exchange capacity (Fig. 2f-h), and developed a severe inflammatory response (significant elevation in levels of IFN $\gamma$, IL-1 $\alpha$, IL-1 $\beta$, IL6, IL-8, TNFa in the bronchoalveolar lavage (BAL) fluid, $p<$ 0.001; Fig. 2i), consistent with previously described effects of large volume aspiration ${ }^{25}$. Significantly more inflammatory cells, including lymphocytes, polymorphonuclear cells, and mast cells, were observed in BAL fluid from injured lungs compared to BAL fluid from control lungs (Fig. 2j; Supplementary Fig. 1j).

Histologic and immunohistochemical evaluation revealed dramatic inflammatory response, cellular derangement, and peribronchial edema, with localized regions of alveolar hemorrhage, alveolar edema, pervasive infiltration of leukocytes (Fig. 2k; Supplementary Fig. 1e-h), perivascular edema, vascular intimal thickening, and extravasation of inflammatory cells (Fig. 2l) in injured lungs. Immunostaining for caspase 3, a marker of early apoptosis, showed numerous apoptotic cells throughout injured lungs $6 \mathrm{~h}$ after gastric aspiration, while apoptotic cells were very rarely observed in control lungs (Fig. 2m). Pathologic assessments were performed in a randomized and blinded fashion according to a previously established protocol ${ }^{24,26,27}$ (Supplementary Table 6) to obtain lung injury scores of injured and control lungs at baseline and $6 \mathrm{~h}$ after gastric aspiration. Injured lungs received significantly higher injury scores than control lungs across all injury categories (Fig. 2n; Supplementary Fig. 1k).

Therapeutic intervention in lungs during cross-circulation. Bronchoalveolar lavage (performed with a flexible video bronchoscope, Supplementary Movie 4), surfactant replacement, and alveolar recruitment maneuvers were conducted according to a specified sequence of interventions and functional assessments (Fig. 1b). Analysis of BAL fluid from injured lungs demonstrated significant decreases in turbidity, 
a

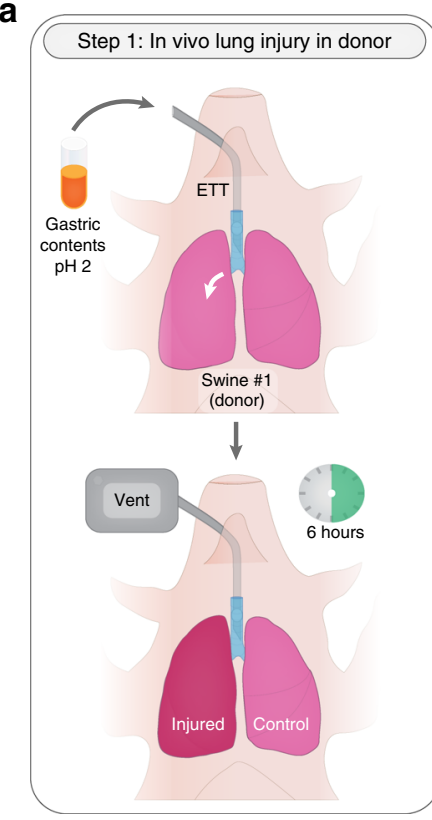

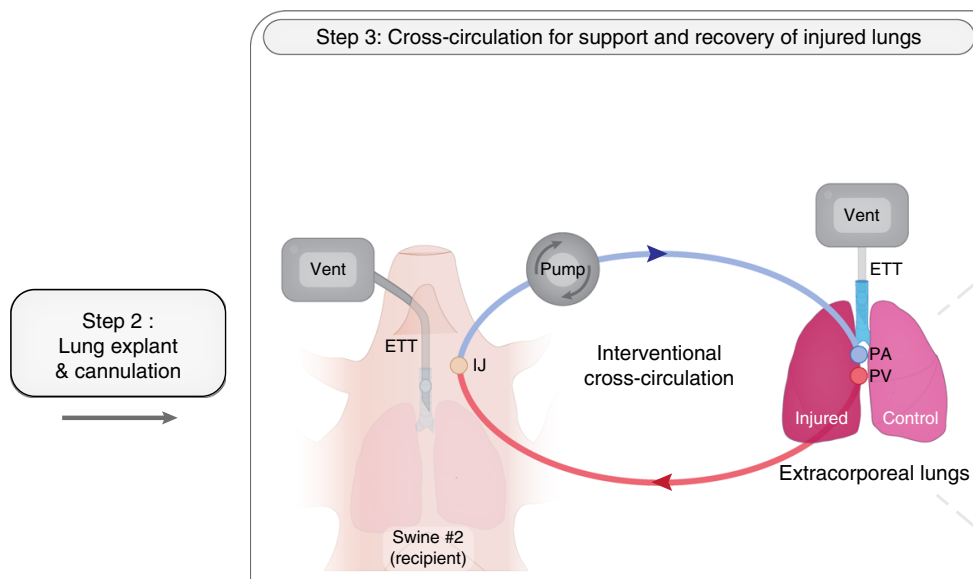

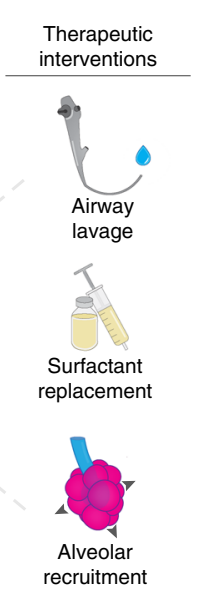

Lung diagnostics

- BAL fluid (cytopathology, cytokines, exosomes) - Histology (optical, fluorescence, electron microscopy)

- Functional analysis (gas exchange, compliance) - Non-invasive imaging (thermography, X-ray, transpleural)

b

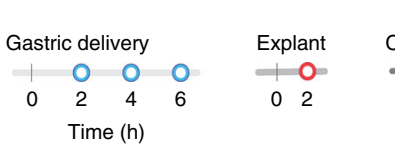

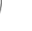
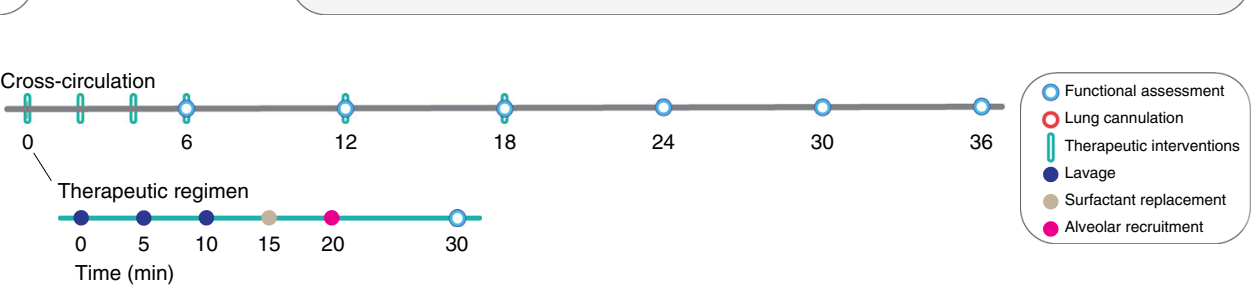

C

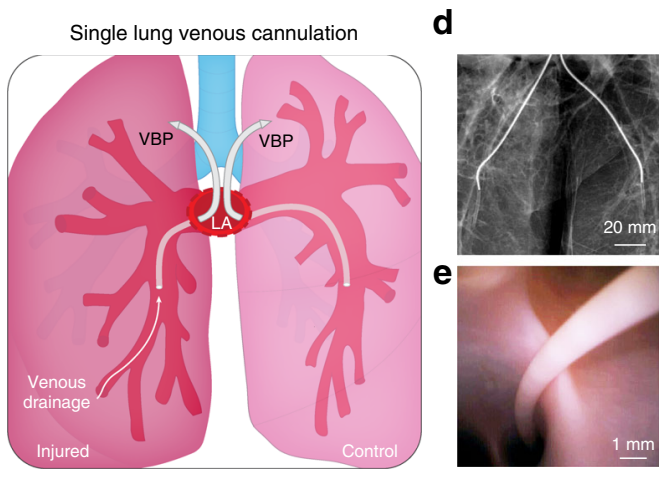

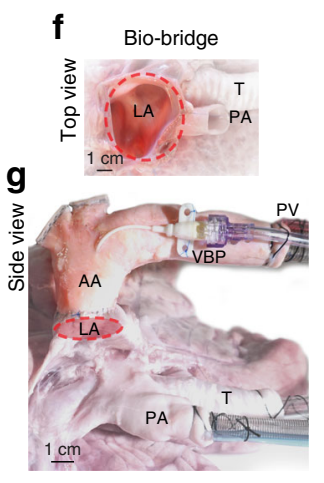

h

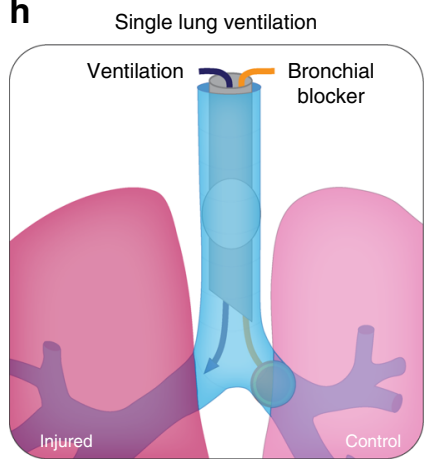

i

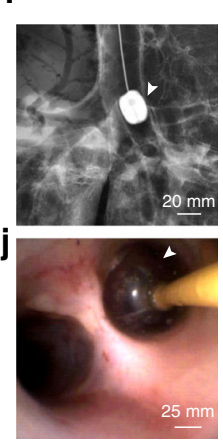

Fig. 1 Experimental overview and technique for single lung functional assessment. a Setup of interventional cross-circulation procedures. Lungs were subjected to injury in vivo by aspiration via bronchoscopic delivery of standardized gastric contents, explanted after $6 \mathrm{~h}$, cannulated, and recovered by cross-circulation. ETT endotracheal tube, IJ internal jugular vein, PA pulmonary artery, PV pulmonary vein. Detailed descriptions and photographs of in vivo injury, lung cannulation procedures, and cross-circulation equipment and setup are available in Supplementary Fig. 1-3 and Supplementary Movie 1.

b Timeline of gastric aspiration injury and lung recovery by therapeutic interventions and cross-circulation. Single lung venous cannulation technique: c Schematic of venous cannulae placed in the left and right pulmonary veins and exiting out of the left atrial (LA) cuff to venous blood ports (VBP), allowing for blood-gas analysis of venous drainage from injured and control lungs. $\mathbf{d}$ X-ray confirming position of cannulae within the left and right pulmonary veins. e Fiber optic image confirming cannulation of a pulmonary vein. Management of pulmonary venous drainage. $\mathbf{f} L A$ cuff after removal of the heart, shown in relation to the pulmonary artery (PA) and trachea ( $T$ ). $\mathbf{g}$ Lungs fully cannulated prior to cross-circulation. Aortic arch (AA) serving as a bio-bridge between the LA cuff and the pulmonary venous (PV) drainage cannula. Placed and secured through the bio-bridge are selective VBPs (only one VBP is visualized on this side view). Single lung ventilation technique: $\mathbf{h}$ Schematic of bronchial blocker placed in one main stem bronchus, enabling isolated ventilation and data acquisition from a single lung. i X-ray confirming position of bronchial blocker within main stem bronchus with balloon inflated (arrow). $\mathbf{j}$ Bronchoscopy showing balloon inflated (arrow) and secured in main stem bronchus of unventilated lung

sediment (gastric contents, airway secretions, and debris), proteinaceous contents, and cell counts $(0 \mathrm{~h}, 1816 \pm 244$ cells; $36 \mathrm{~h}, 120 \pm 31$ cells; $p<0.001)$ over $36 \mathrm{~h}$ of cross-circulation (Fig. 3a-c). The concentration of pepsin in BAL fluid from injured lungs also decreased significantly over $36 \mathrm{~h}$ of crosscirculation $\left(0 \mathrm{~h}, 111.9 \pm 43.2 \mathrm{ng} \mathrm{mL}^{-1}\right.$; $36 \mathrm{~h}, 5.8 \pm 3.9 \mathrm{ng} \mathrm{mL}^{-1}$; $p<0.001)$, while the $\mathrm{pH}(0 \mathrm{~h}, 4.5 \pm 0.2)$ normalized to the $\mathrm{pH}$ of BAL fluid from control lungs $(5.5 \pm 0.1)$ by $12 \mathrm{~h}$ of crosscirculation (Fig. 3d, e). Total protein in BAL fluid from injured lungs gradually decreased over $36 \mathrm{~h}$ of cross-circulation $(0 \mathrm{~h}$, $1.51 \pm 0.29 \mathrm{mg} \mathrm{mL}^{-1} ; 36 \mathrm{~h}, 1.04 \pm 0.16 \mathrm{mg} \mathrm{mL}^{-1}$ ) but remained consistently elevated compared to BAL fluid from control 


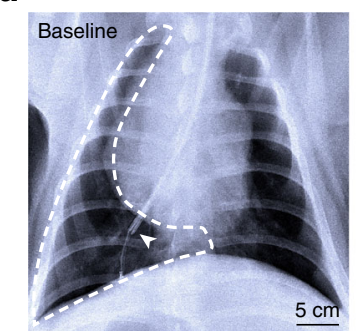

b

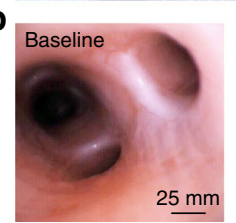

Donor response to gastric aspiration
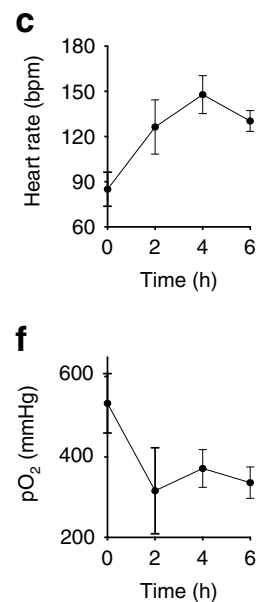
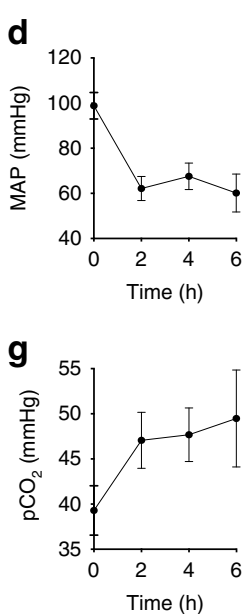
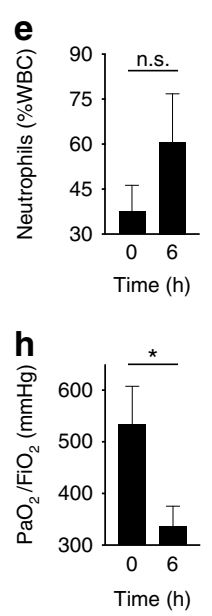

i

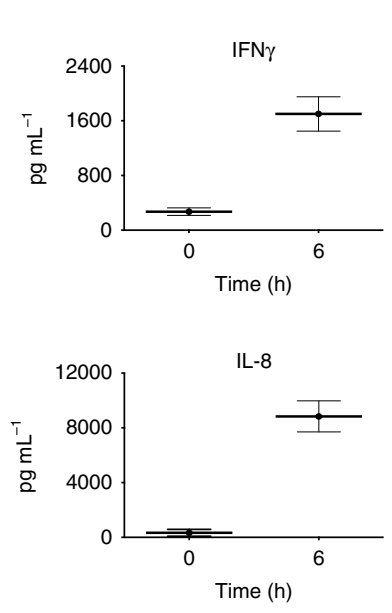

Airway response to gastric aspiration
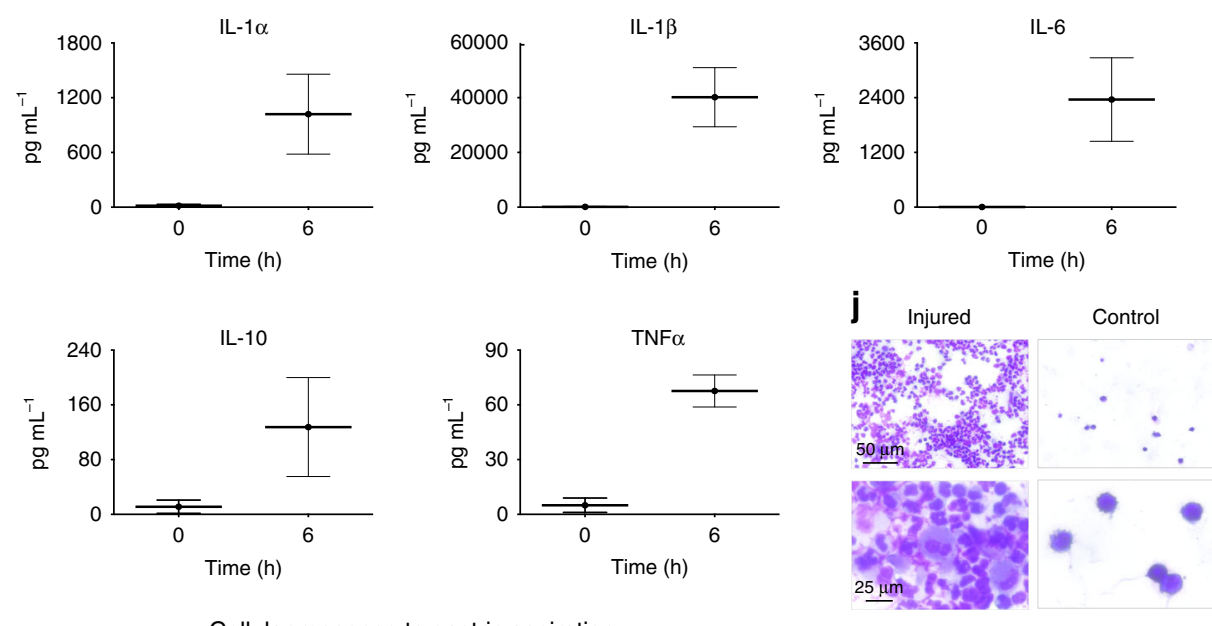

Cellular response to gastric aspiration
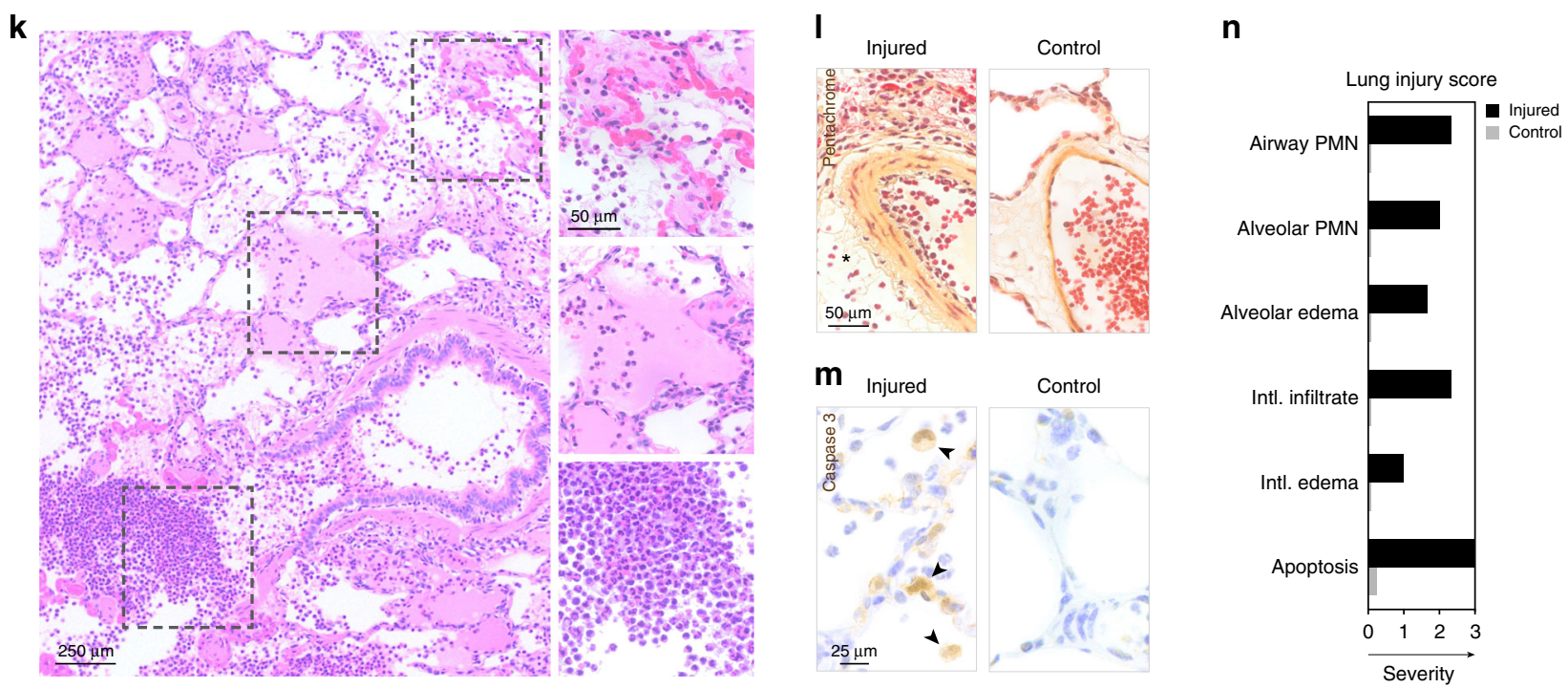

lungs $\left(0 \mathrm{~h}, 0.25 \pm 0.13 \mathrm{mg} \mathrm{mL}^{-1} ; 36 \mathrm{~h}, 0.28 \pm 0.18 \mathrm{mg} \mathrm{mL}^{-1}\right.$, Fig. $3 f$ ).

Interestingly, the concentration of exosomes in BAL fluid from injured lungs was significantly elevated at $12 \mathrm{~h}$ of crosscirculation (injured, $8.96 \times 10^{15} \pm 2.24 \times 10^{15}$ particles $\mathrm{mL}^{-1}$; control, $4.88 \times 10^{14} \pm 3.90 \times 10^{14}$ particles $\left.\mathrm{mL}^{-1} ; p<0.01\right)$ but

trended downward between 12 and $36 \mathrm{~h}$ of cross-circulation (Fig. 3g). Correlation between injury/inflammation and exosome concentration has been previously reported in other pathologic conditions $^{28,29}$, and these results suggest that the concentration of exosomes in BAL fluid may serve as a diagnostic marker of extracorporeal lung injury and recovery. Exosomes in BAL fluids 
Fig. 2 In vivo response to gastric aspiration injury. a Donor chest X-ray at baseline and $6 \mathrm{~h}$ after gastric aspiration. Arrow indicates tip of bronchoscope within the injured lung. $\mathbf{b}$ Bronchoscopic analysis of pulmonary airways at baseline, at the time of aspiration, and $6 \mathrm{~h}$ after gastric aspiration. Systemic response of donor following gastric aspiration: c heart rate, $\mathbf{d}$ mean arterial pressure (MAP), and e neutrophils (\% white blood cells (WBC)), Student's $t$ test for $\mathrm{O}$ h versus $6 \mathrm{~h}$, not significant (n.s.). Lung function of donor following aspiration: $\mathbf{f}$ oxygenation $\left(\mathrm{pO}_{2}\right), \mathbf{g}$ ventilation $\left(\mathrm{pCO}_{2}\right)$, and $(\mathbf{h}) \mathrm{PaO}_{2} / \mathrm{FiO}_{2}$ ratio, Student's $t$-test for $0 \mathrm{~h}$ versus $6 \mathrm{~h},{ }^{\star} p<0.05$. i Inflammatory response measured in donor bronchoalveolar lavage (BAL) fluid at baseline and $6 \mathrm{~h}$ after gastric aspiration injury; for all graphs shown, Student's $t$-test for $0 \mathrm{~h}$ versus $6 \mathrm{~h}, p<0.001$. j Kwik-Diff staining of BAL fluid smears from injured and control lungs $6 \mathrm{~h}$ after gastric aspiration. $\mathbf{k} \mathrm{H} \& \mathrm{E}$ staining of injured lung $6 \mathrm{~h}$ after gastric aspiration, with high magnification insets demonstrating regions of alveolar hemorrhage, alveolar edema and cellular exudate, and exudate of leukocytes into alveoli. I Pentachrome staining $6 \mathrm{~h}$ after gastric aspiration. Perivascular edema and infiltration noted in the injured lung (star). $\mathbf{m}$ Caspase 3 immunostaining, a marker of early apoptosis (arrows), $6 \mathrm{~h}$ after gastric aspiration. n Lung injury score obtained at $6 \mathrm{~h}$ after gastric aspiration. PMN polymorphonuclear leukocytes. All graphs represent data from $n=8$ lungs. All values represent mean \pm standard deviation
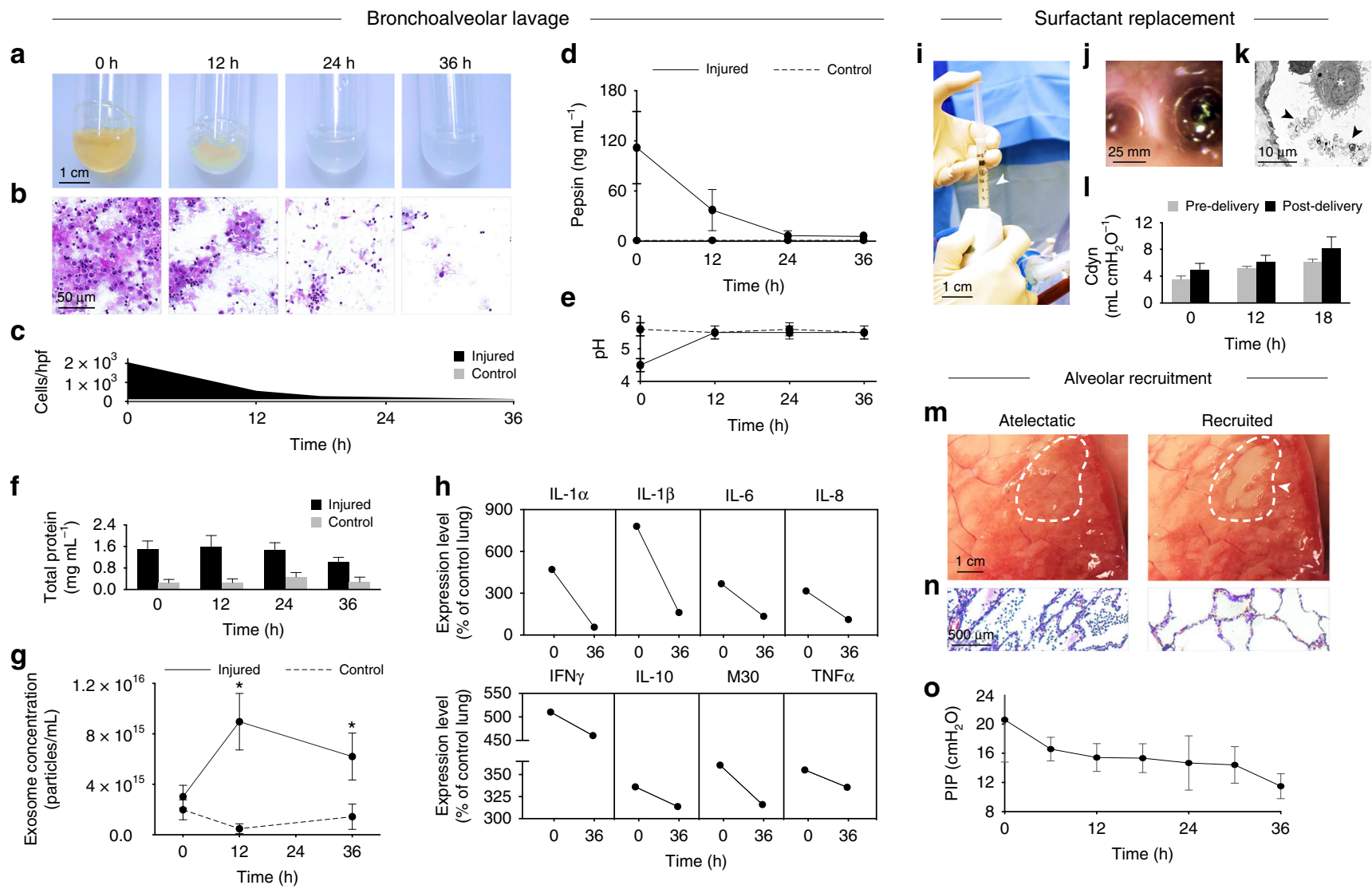

Fig. 3 Therapeutic interventions in injured lungs during cross-circulation. a Gross imaging of BAL fluid samples collected from injured lungs over $36 \mathrm{~h}$ of cross-circulation. b Periodic acid-Schiff staining of BAL fluid smears obtained from injured lungs. c Quantification of cells in BAL fluid samples. d Pepsin concentration, e $\mathrm{pH}, \mathbf{f}$ total protein, and $\mathbf{g}$ exosome concentration in BAL fluid samples over $36 \mathrm{~h}$ of cross-circulation, Student's $t$-test for Injured versus Control, ${ }^{*} p<0.001$. h Mean cytokine expression levels in injured lungs, normalized as a percent of mean levels in control lungs, measured in BAL fluid between initiation of cross-circulation $(\mathrm{O} h)$ and conclusion of cross-circulation $(36 \mathrm{~h})$. Cytokine concentrations (mean \pm standard deviation) throughout $36 \mathrm{~h}$ of cross-circulation in Supplementary Fig. 5c. Surfactant replacement: i Bronchoscopic delivery of therapeutic surfactant into injured lung (arrow). j Bronchoscopic image demonstrating therapeutic surfactant delivered into airways within the injured lung. $\mathbf{k}$ Transmission electron micrograph (TEM) demonstrating abundant surfactant (arrows) within alveoli following delivery. Alveolar macrophage (star) and adjacent lymphocyte observed within the same alveoli. I Dynamic compliance (Cdyn) of injured lungs pre- and post-delivery of therapeutic surfactant over the first $18 \mathrm{~h}$ of cross-circulation. $\mathbf{m}$ Alveolar recruitment: before recruitment (atelectatic) and after recruitment (recruited). Arrow and dotted line indicates a region of recruitment. $\mathbf{n}$ Silver reticulin staining of collapsed consolidated lung, and recruited lung with open alveoli. o Peak inspiratory pressure (PIP) of injured lungs throughout $36 \mathrm{~h}$ of cross-circulation. All graphs represent data from $n=8$ lungs. All values represent mean \pm standard deviation

from injured and control lungs had comparable mean diameters and size distributions (Supplementary Fig. $4 d-f$ ). In parallel, the effect of interventional cross-circulation on the inflammatory response in injured lungs was analyzed by quantification of cytokines in BAL fluid. GM-CSF, IFN $\gamma$, IL-1 $\alpha$, IL-1 $\beta$, IL-2, IL-4, IL-6, IL-8, IL-12, IL-18, M30, and TNFa all decreased over $36 \mathrm{~h}$ of cross-circulation (Fig. 3h, Supplementary Table 9, Supplementary Fig. 4c).
Following airway lavage, exogenous surfactant was delivered via video bronchoscopy into each lobe of injured lungs (Fig. 3i, j; Supplementary Fig. 8g), and was observed advancing distally through the airway tree during positive pressure ventilation, consistent with the transport and deposition of liquid plugs through small airways ${ }^{30,31}$ (Supplementary Movie 5). Transmission electron microscopy confirmed the presence of abundant surfactant in distal airspaces (Fig. 3k; Supplementary Fig. 8h). 
Dynamic compliance of injured lungs consistently increased after surfactant delivery and throughout the duration of interventional cross-circulation (Fig. 31). Following surfactant replacement, alveolar recruitment maneuvers were performed by adjusting ventilator settings to recruit additional lung volumes ${ }^{32,33}$. Gross imaging of the lung surface (Fig. 3m, Supplementary Movie 6) and histologic analysis (Fig. $3 \mathrm{n}$ ) showed that atelectatic regions throughout the injured lung were successfully recruited over $36 \mathrm{~h}$ of interventional cross-circulation. Peak inspiratory pressures (PIP) during standard ventilation gradually decreased from 20.6 $\pm 5.8 \mathrm{cmH}_{2} \mathrm{O}$ to $11.5 \pm 1.7 \mathrm{cmH}_{2} \mathrm{O}$ over $36 \mathrm{~h}$ of cross-circulation (Fig. 3o), consistent with lung recovery and recruitment of additional lung volumes.

Functional assessment of lungs during cross-circulation. Lung weight significantly decreased over $18 \mathrm{~h}(0 \mathrm{~h}, 0.75 \pm 0.06 \mathrm{~kg} ; 18 \mathrm{~h}$, $0.61 \pm 0.05 \mathrm{~kg}, p<0.05)$ and remained stable through $36 \mathrm{~h}(18 \mathrm{~h}$, $0.61 \pm 0.05 \mathrm{~kg} ; 36 \mathrm{~h}, 0.54 \pm 0.06 \mathrm{~kg}, p>0.05)$ of cross-circulation (Supplementary Fig. 5e). Trans-pulmonary pressure gradients (TPG) were tightly maintained at $5-15 \mathrm{mmHg}$ throughout crosscirculation (Supplementary Fig. 3j). Significant improvements of pressure-volume (PV) loops and dynamic compliance of injured lungs were observed throughout $36 \mathrm{~h}$ of cross-circulation. Initially, injured lungs displayed significant derangement of PV loops (PIP: $28.7 \pm 4.4 \mathrm{cmH}_{2} \mathrm{O}$, volume: $67.9 \pm 16.3 \mathrm{~mL}$; injured, $0 \mathrm{~h}$, red loop, Fig. 4a) and significantly reduced dynamic compliance (injured, $2.83 \pm 1.86 \mathrm{~mL} \mathrm{cmH} \mathrm{O}^{-1}$; control, $23.03 \pm 1.84 \mathrm{~mL}$ $\mathrm{cmH}_{2} \mathrm{O}^{-1} ; p<0.01$; Fig. $4 \mathrm{~b}$ ). After $36 \mathrm{~h}$ of interventional crosscirculation, injured lungs recovered normal hysteresis (PIP: 17.9 $\pm 3.6 \mathrm{cmH}_{2} \mathrm{O}$, volume: $186.6 \pm 23.8 \mathrm{~mL}$; injured, $36 \mathrm{~h}$, blue loop, Fig. 4a) similar to that of control lungs (PIP: $14.5 \pm 2.1 \mathrm{cmH}_{2} \mathrm{O}$, volume: $227.5 \pm 12.4 \mathrm{~mL}$; control, $36 \mathrm{~h}$, dark blue loop, Fig. $4 \mathrm{a}$ ). Injured lungs maintained on $40 \% \mathrm{FiO}_{2}$ had an average initial $\mathrm{PaO}_{2}$ of $36 \pm 28 \mathrm{mmHg}$, corresponding to an average initial $\mathrm{PaO}_{2} /$ $\mathrm{FiO}_{2}$ ratio of $90 \pm 70 \mathrm{mmHg}$, and demonstrated steady improvements in gas exchange function through the first $12 \mathrm{~h}$ of crosscirculation (12 h, $\mathrm{PaO}_{2}: 257 \pm 57 \mathrm{mmHg}, \mathrm{PaO}_{2} / \mathrm{FiO}_{2}: 643 \pm 144$ $\mathrm{mmHg}$ ). Control lungs maintained an average $\mathrm{PaO}_{2}$ of $309 \pm 57$ $\mathrm{mmHg}$, and $\mathrm{PaO}_{2} / \mathrm{FiO}_{2}$ of $773 \pm 142 \mathrm{mmHg}$ (Fig. 4c).

Injured and control lungs demonstrated differential capacities to exchange gas. Response to increasing minute ventilation by $100 \%$ and increasing $\mathrm{FiO}_{2}$ from 40 to $100 \%$ was demonstrated by differences $(\Delta)$ in baseline and challenge levels of pulmonary artery and vein hemogases (Fig. 4h), $\Delta \mathrm{pO}_{2}$ (oxygenation, Fig. $4 \mathrm{~d}, \mathrm{f}$ ) and $\Delta \mathrm{pCO}_{2}$ (ventilation, Fig. $4 \mathrm{e}, \mathrm{g}$ ). Notably, injured lungs achieved $\Delta \mathrm{pO}_{2}$ values of control lungs after $12 \mathrm{~h}$ of crosscirculation (Fig. $4 \mathrm{~d}$ ) and $\Delta \mathrm{pCO}_{2}$ values of control lungs after $30 \mathrm{~h}$ of cross-circulation (Fig. $4 \mathrm{e}$ ).

Recovery of injured lungs during cross-circulation. Injured lungs had sizable areas of consolidation and discoloration suggestive of pulmonary edema and hemorrhage that were reduced after $18 \mathrm{~h}$ and resolved after $36 \mathrm{~h}$ of cross-circulation (Fig. 5a; Supplementary Fig. 5a-c). Radiography revealed severe unilateral injury with marked lobar and multi-focal opacities and the presence of air bronchograms throughout injured lungs (Fig. 5b; Supplementary Fig. 5d), while contralateral control lungs maintained normal radiographic appearance. Progressive clearance was observed throughout interventional cross-circulation, with recovery of aerated lung volume in injured lungs after $36 \mathrm{~h}$.

Thermography was used to assess global and local changes in lung surface temperatures throughout $36 \mathrm{~h}$ of cross-circulation. Injured and contralateral control lungs were rewarmed in the normothermic organ chamber and achieved steady state surface temperatures within $30 \mathrm{~min}$ of reperfusion
(Supplementary Fig. 31, m; Supplementary Movie 3). Notably, through $18 \mathrm{~h}$ of cross-circulation, injured lungs showed more heterogeneous and elevated surface temperatures compared to contralateral regions of control lungs (Fig. 5c; Supplementary Fig. 8a). Regions of injured lungs with elevated surface temperatures correlated with areas of consolidation and hemorrhage, consistent with perfused parenchyma with diminished ventilation. After $36 \mathrm{~h}$ of cross-circulation, no significant differences in surface temperatures were observed across injured lungs or between injured and control lungs (Fig. $5 \mathrm{c}$, d; Supplementary Fig. 8a, b). These results suggest that surface thermography is a useful diagnostic imaging modality for noninvasive assessment of extracorporeal lung recovery.

Lung surface regions (diameter: $8 \mathrm{~mm}$ ) were topically cooled by contact with a hypothermic probe (Fig. 5f; Supplementary Fig. 8c, d). In control lungs, surface temperatures returned to baseline within $20 \mathrm{~s}$ (Supplementary Fig. 8e), while injured lungs consistently demonstrated protracted recovery, requiring more than $45 \mathrm{~s}$ to return to baseline (Fig. 5g; Supplementary Movie 7). Differences in surface temperatures between topically cooled regions and surrounding areas were quantified using video thermography and image analysis software. Temperature differences decreased exponentially over time (Fig. 5e, Supplementary Fig. 8f), with time constants for injured lungs $(0 \mathrm{~h}, \tau=71.4 \mathrm{~s}$; $12 \mathrm{~h}, \tau=39.1 \mathrm{~s} ; 24 \mathrm{~h}, \tau=38.5 \mathrm{~s} ; 36 \mathrm{~h}, \tau=36.2 \mathrm{~s})$ gradually decreasing to values of time constants for control lungs $(0 \mathrm{~h}, \tau$ $=33.3 \mathrm{~s} ; 12 \mathrm{~h}, \tau=30.3 \mathrm{~s} ; 24 \mathrm{~h}, \tau=32.2 \mathrm{~s} ; 36 \mathrm{~h}, \tau=30.3 \mathrm{~s}$ ). These results suggest that in addition to global surface thermography, regional thermodynamic imaging may be a useful diagnostic tool for real-time, non-invasive assessment of extracorporeal organs to identify and monitor injury resulting in ventilation-perfusion mismatch.

The airways of injured lungs were inflamed and clearly distinguishable from airways of control lungs. After $18 \mathrm{~h}$ of interventional cross-circulation, airway bronchoscopy showed reduced erythema of injured airways, with resolving inflammation of the bronchial mucosa (Fig. 5k). After $36 \mathrm{~h}$, only minimal residual inflammation was observed in airways of injured lungs. Histologic analyses of control lungs revealed normal tissue histomorphology with intact columnar bronchial epithelium and alveolar architecture, and no evidence of pulmonary edema or inflammation throughout $36 \mathrm{~h}$ of cross-circulation (Supplementary Fig. 5f, 7c), consistent with previous studies wherein our group demonstrated robust maintenance of healthy lungs over $36 \mathrm{~h}$ of cross-circulation support ${ }^{24}$.

In contrast, injured lungs displayed pervasive inflammation and disruption of the lung parenchyma, with extensive airway congestion, interstitial and alveolar edema, hemorrhage, leukocyte infiltration, leakage of lymphatic and plasma proteins, and cellular debris (Fig. 5h, $0 \mathrm{~h}$ ). Scanning and transmission electron microscopy showed severe tissue derangement, exudation, and loss of stereotypical saccular structures (Fig. 5i, 0 h), with alveolar spaces containing particulate debris, extravascular erythrocytes consistent with alveolar hemorrhage, alveolar septal thickening, neutrophil extravasation, and an impaired blood-gas barrier with severely compromised respiratory epithelium, disruption of type I pneumocyte cell membranes, and atypical cytomorphology of type II pneumocytes (Fig. 5j, 0 h; Supplementary Fig. 7b). Markers of injury were drastically reduced after $18 \mathrm{~h}$ of interventional cross-circulation, with evidence of partial restoration of the alveolar epithelial lining, and complete resolution by $36 \mathrm{~h}$ of interventional cross-circulation (Fig. 5h-j, $36 \mathrm{~h}$; Supplementary Fig. 7b).

Pathologic assessment of injured and control lungs was performed in a randomized blinded fashion to obtain categorical and composite lung injury scores according to a previously 
a

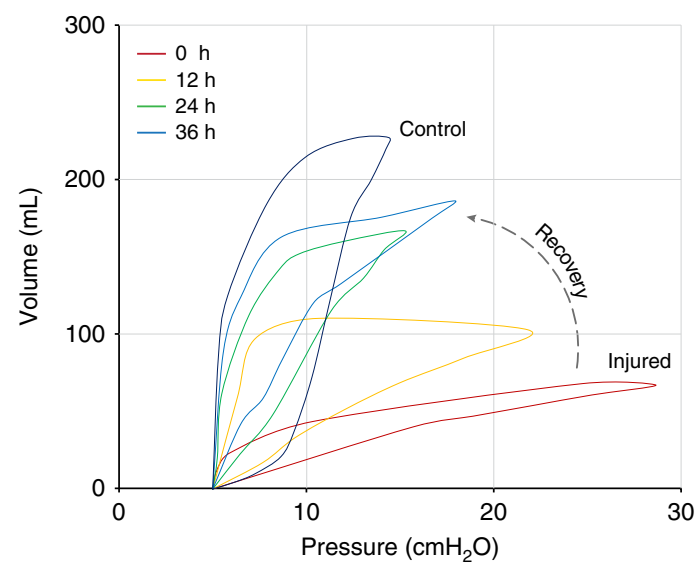

b
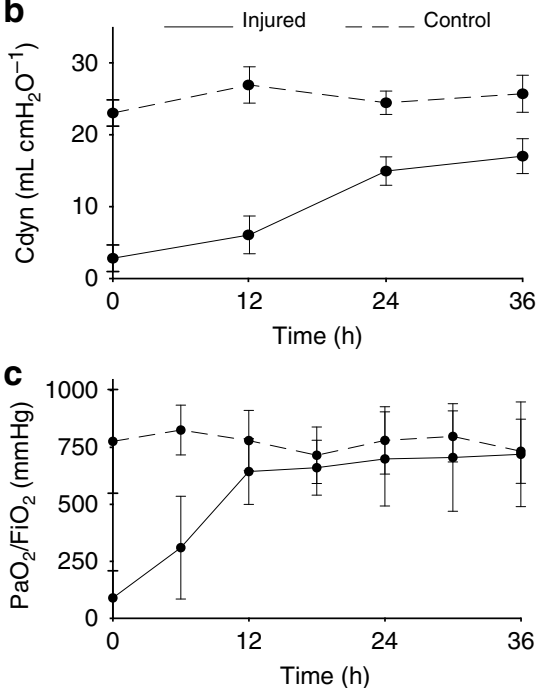
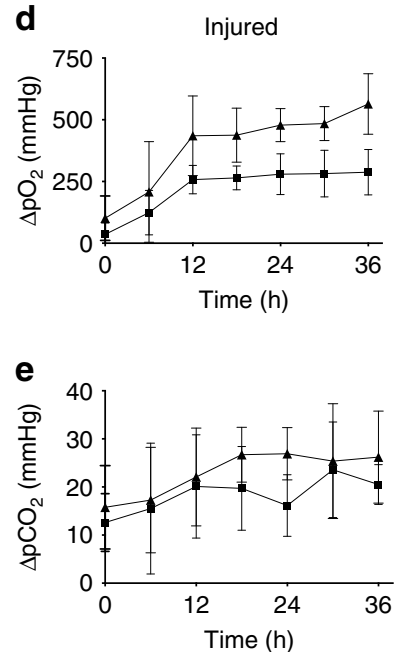

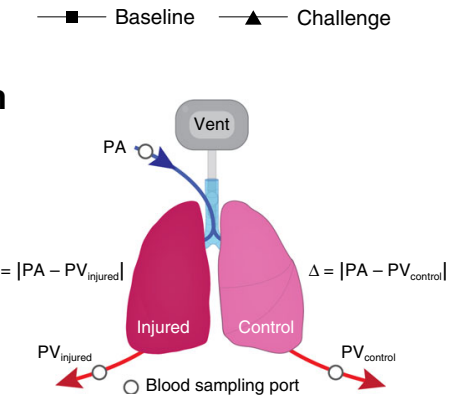

Lung performance challenge:

1) Obtain baseline hemogases ( $\left.P A, P V_{\text {injured }}, P V_{\text {control }}\right)$ 2) Calculate baseline $\triangle \mathrm{pO}_{2}$ and $\triangle \mathrm{pCO}_{2}$

3) Initiate challenge (min. vent $\left.x 2, \mathrm{FiO}_{2} 100 \%\right) \times 10 \mathrm{~min}$

4) Obtain challenge hemogases ( $\left.P A, P V_{\text {injured }}, \mathrm{PV}_{\text {control }}\right)$ 5) Calculate challenge $\triangle \mathrm{pO}_{2}$ and $\triangle \mathrm{pCO}_{2}$ f
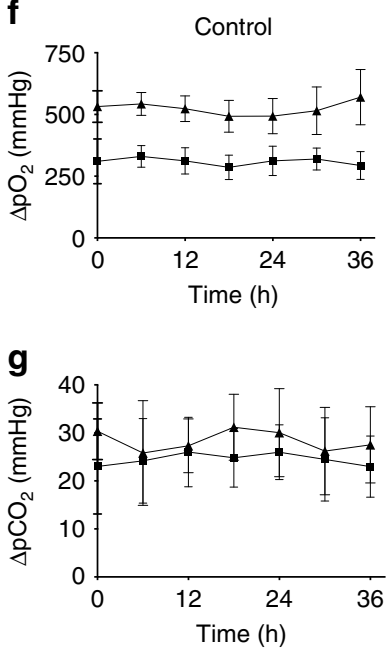

Fig. 4 Extracorporeal lung performance and functional recovery. a Pressure-volume (PV) loops: injured lungs demonstrating initial derangement in PV

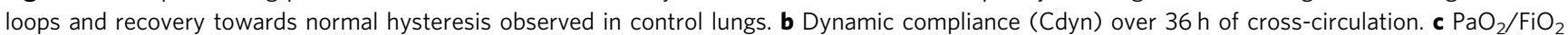
over $36 \mathrm{~h}$ of cross-circulation. Responses in $\Delta \mathrm{pO}_{2}$ and $\Delta \mathrm{pCO}_{2}$ to 10 min lung performance challenges throughout $36 \mathrm{~h}$ of cross-circulation in $\mathbf{d}$, e injured and $\mathbf{f}, \mathbf{g}$ control lungs. $\mathbf{h}$ Schematic depicts arterial and single lung venous blood sample collection and performance challenge protocol. All graphs represent data from $n=8$ lungs. All values represent mean \pm standard deviation

established scoring rubric ${ }^{24}$ (Supplementary Table 6). Lung injury was evaluated by quantification of polymorphonuclear (PMN) cells in airways and alveoli, early and late apoptotic cells, interstitial and alveolar edema, and interstitial infiltrates (Supplementary Fig. 6). Lung injury scores trended downward in all categories over $36 \mathrm{~h}$ of interventional cross-circulation (Fig. 5l; Supplementary Fig. 5g). Apoptosis was the lung injury category with the most significant reduction in injury score, decreasing from 3.0 at initiation to 0.3 after $36 \mathrm{~h}$ of interventional crosscirculation. Control lungs showed no changes in categorical or composite lung injury scores across $36 \mathrm{~h}$ of cross-circulation (Fig. 5l; Supplementary Fig. 5h). The difference in composite lung injury scores between injured and control lungs decreased throughout $36 \mathrm{~h}$ of cross-circulation from 12.1 to 5.7 (Supplementary Fig. 5i), suggesting a high degree of lung recovery.

Regeneration of injured lungs during cross-circulation. Silver reticulin staining of injured lungs demonstrated dense cellular debris and mucus within the bronchial lumen, peribronchial edema, and consolidation of alveoli, consistent with severe acute lung injury and disruption of the pulmonary epithelium. Alcian blue staining showed abundant trans-epithelial migration of neutrophils into the airway lumen through pseudostratified airway epithelium, and apparent loss of secretory vesicles containing mucus in goblet cells. Periodic acid-Schiff and pentachrome staining showed damaged, discontinuous columnar epithelium with exposed basement membrane, inflammatory cells, and loss of airway cilia (Fig. 6a). After $36 \mathrm{~h}$ of cross-circulation, conducting airways of injured lungs exhibited resolution of edema and inflammation, clearance of luminal debris consistent with the histologic appearance of proximal airways in control lungs, trace evidence of neutrophil infiltration, presence of mucus-filled granules in goblet cells, and intact columnar epithelium with reconstituted ciliated brush border (Fig. 6a). In the respiratory zone of injured lungs, retention of structural fibers in the alveolar extracellular matrix, clearance of alveolar edema and inflammatory cell infiltration, absence of extravascular erythrocytes, reduced perivascular edema, and restored vascular histomorphology were observed (Fig. 6b, c).

Injured lungs also contained significantly more neutrophils than control lungs, with significantly greater myeloperoxidase 
a
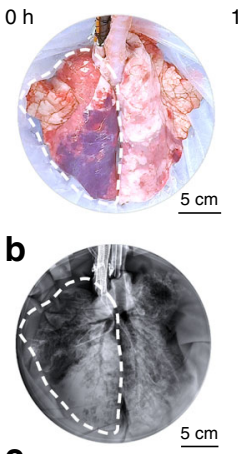

C

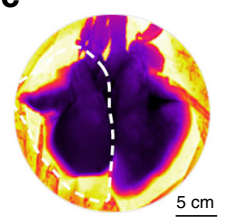

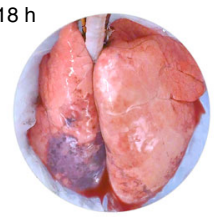
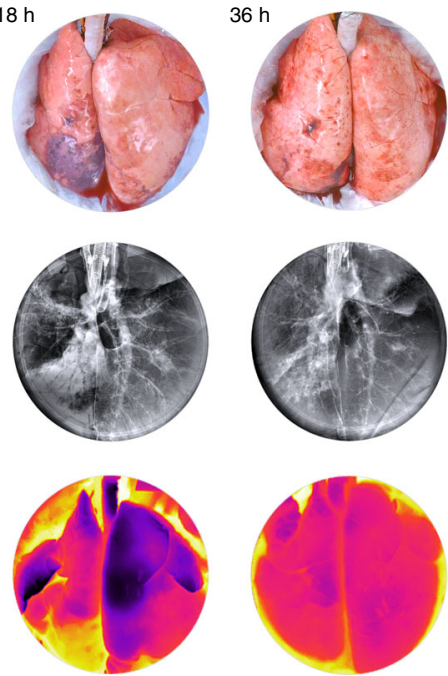
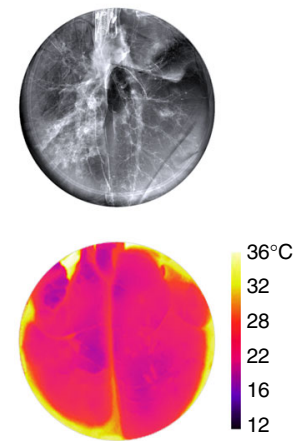

h
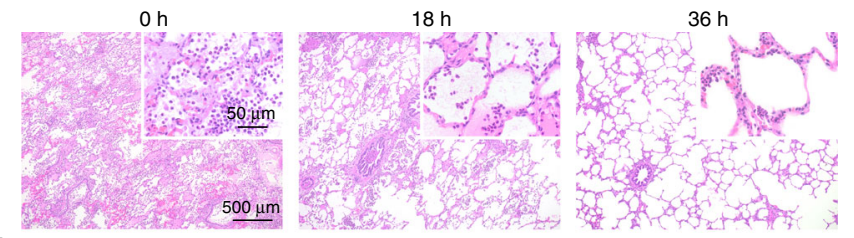

i
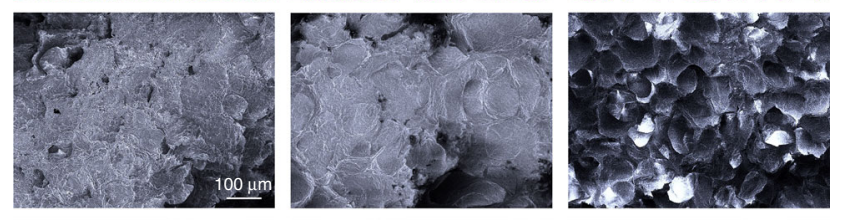

j
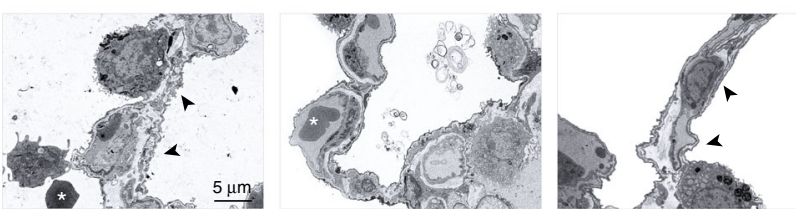

k Injured
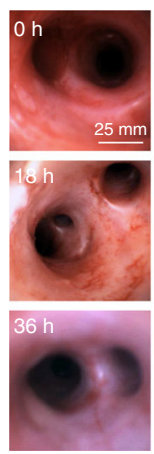

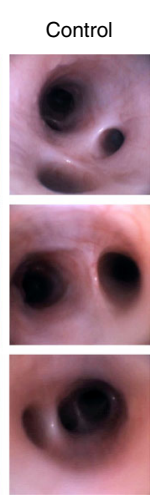

I

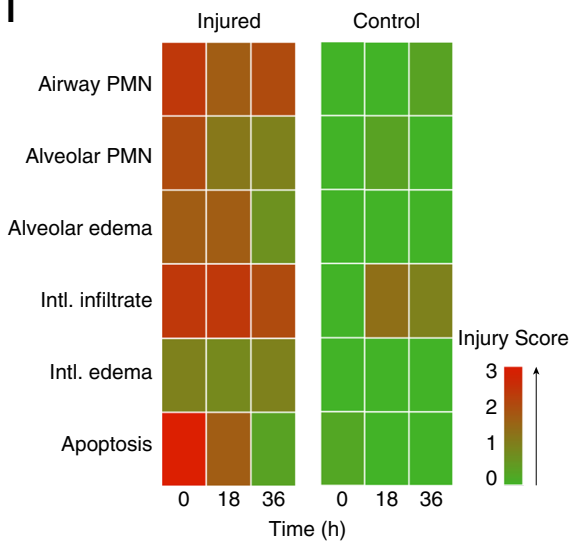

Fig. 5 Multi-scale analysis of extracorporeal lung recovery. Macroscopic appearance: a photographic, $\mathbf{b}$ radiographic, and $\mathbf{c}$ thermographic analysis of lungs throughout $36 \mathrm{~h}$ of cross-circulation. Dotted line indicates the injured lung. d Average recorded surface temperature of injured and control lungs throughout $36 \mathrm{~h}$ of cross-circulation. e Difference in temperature $(\Delta)$ between hypothermic lung region and average temperature of surrounding pleura over $45 \mathrm{~s}$ of rewarming. Surface cooling and rewarming challenge: $\boldsymbol{f}$ thermographic image of lungs following contact with a conductive hypothermic probe (arrow), and $\mathbf{g}$ time lapse sequence of injured and control lungs during rewarming at $0,12,24$, and $36 \mathrm{~h}$ of cross-circulation. Microscopic appearance: $\mathbf{h}$ $\mathrm{H} \&$ E staining, i scanning electron microscopic, and $\mathbf{j}$ transmission electron microscopic analysis of injured lungs throughout $36 \mathrm{~h}$ of cross-circulation. Type I alveolar epithelial cell membranes disrupted at $\mathrm{O}$ h and restored at $36 \mathrm{~h}$ (arrows). Red blood cells (stars) shown in the alveolar space at $\mathrm{O} \mathrm{h}$ and within a capillary at $18 \mathrm{~h}$. k Bronchoscopic analysis of airways in injured and control lungs throughout $36 \mathrm{~h}$ of cross-circulation. I Lung injury scoring of injured and control lungs at 0,18 , and $36 \mathrm{~h}$ of cross-circulation. All graphs represent data from $n=8$ lungs. All values represent mean \pm standard deviation

(MPO) activity in injured lung parenchyma (Fig. 6d, e). By $18 \mathrm{~h}$ of cross-circulation, neutrophil numbers and MPO activity were significantly decreased and maintained within normal ranges through $36 \mathrm{~h}$ of cross-circulation, consistent with histologic evaluations (Fig. 6a-c). Additionally, the number of $\mathrm{CD}_{163}{ }^{+}$ macrophages in injured lungs decreased to normal (control) levels by $36 \mathrm{~h}$ of cross-circulation $(p<0.001$, Fig. $6 \mathrm{f})$. Over the same period, the high initial levels of caspase 3 and TUNEL significantly decreased, and apoptotic cells were rarely observed in injured lungs, indicating clearance of dying and dead cells (Fig. 6g; Supplementary Fig. 6a, b). Interestingly, mitochondrial activity showed no significant differences in metabolism of injured or control lungs during $36 \mathrm{~h}$ of cross-circulation support (Fig. 6h).

As aspiration of gastric contents incurs a severe chemical injury to the respiratory epithelium, especially to the type I pneumocytes that comprise the majority of the alveolar surface ${ }^{34}$, the integrity of the alveolar epithelium was assessed. Expression of aquaporin
5 , an integral membrane protein that functions as a water channel and marker of type I pneumocytes, was largely lost following aspiration of gastric contents but recovered during interventional cross-circulation (Fig. 6i; Supplementary Fig. 7a). Initially, total depletion of epithelial tight junctions (zonula occludens-3, ZO-3) and loss of barrier function was observed in injured lungs, but the number of tight junctions increased by $18 \mathrm{~h}$ of cross-circulation (Fig. 6j; Supplementary Fig. 7d). Expression of surfactant protein C (SPC), a marker of type II pneumocytes, was negligible in injured lungs but became increasingly abundant by $36 \mathrm{~h}$ of crosscirculation, with clear punctate distributions suggesting normal intracellular localization within lamellar bodies (Fig. 6k; Supplementary Fig. 7e). Viability and function of type II pneumocytes after $36 \mathrm{~h}$ of cross-circulation were confirmed by uptake of BODIPY-labeled surfactant protein B (BODIPY-SPB) delivered into distal regions of injured lungs (Fig. $6 \mathrm{~m}$ ).

Regeneration of pulmonary endothelium after aspiration of gastric contents was also assessed by analysis of the injured 

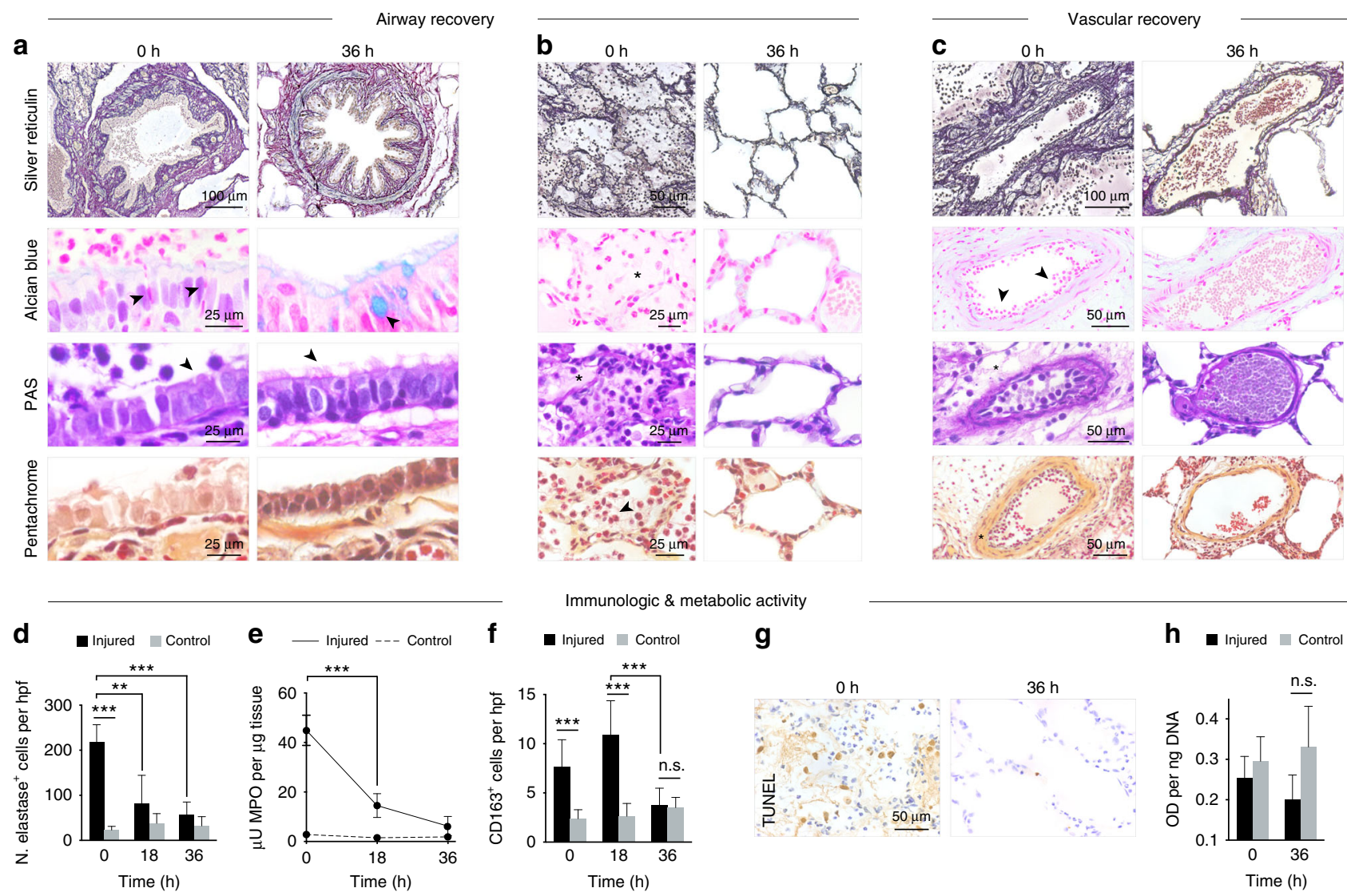

g
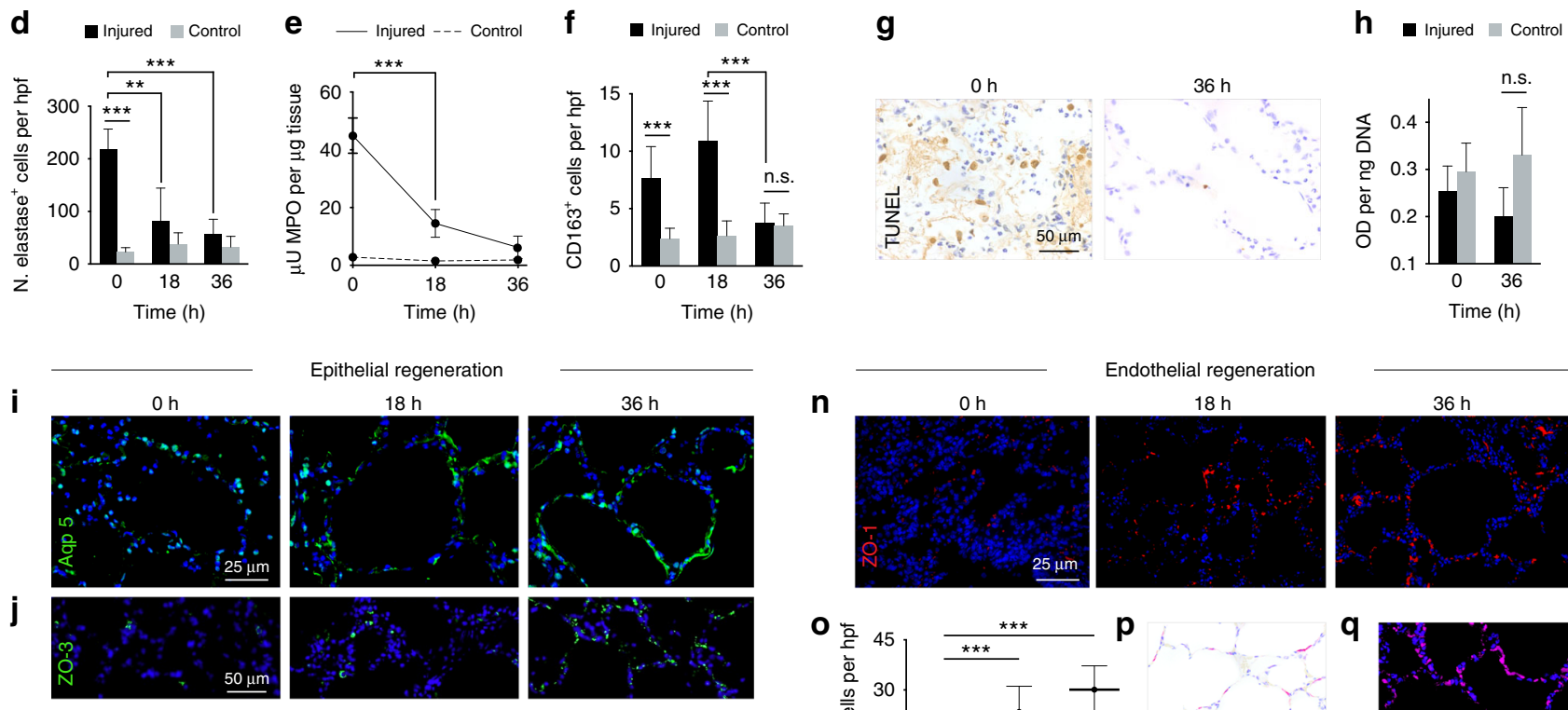

Epithelial regeneration
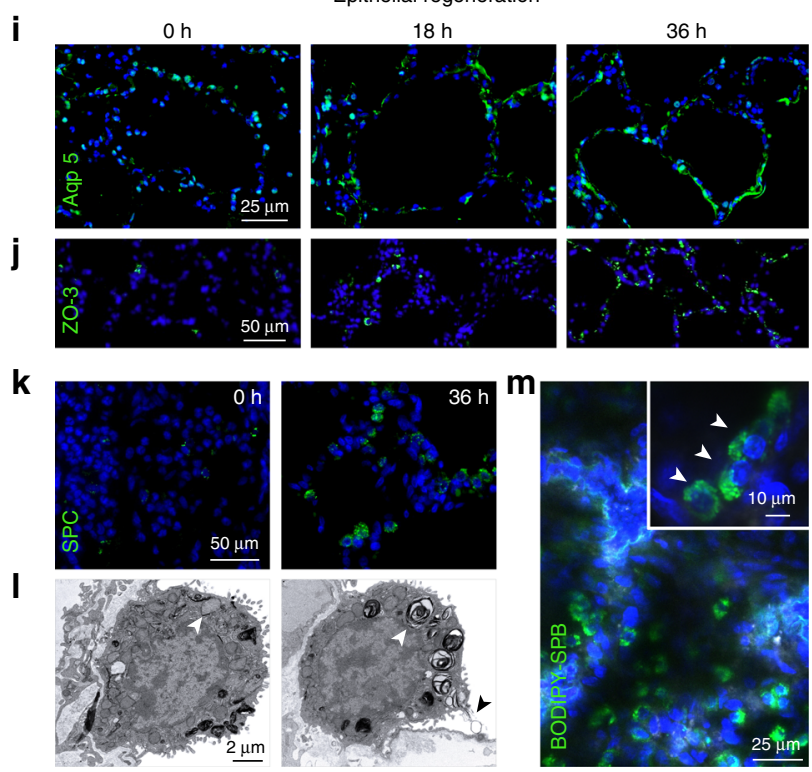

pulmonary vascular network, including microvascular tight and gap junctions. Immunostaining for zonula occludens-1 (ZO-1) revealed dramatic loss of tight junctions throughout the pulmonary microvasculature following injury, but recovery to normal expression levels ${ }^{24}$ after $36 \mathrm{~h}$ of cross-circulation (Fig. 6n; Supplementary Fig. 7d). Quantification of gap junction alpha-1 protein (connexin 43), a component of gap junctions that enables critical intercellular interactions, showed a similar trend of recovery to previously reported expression levels in healthy controls $^{35}$ (Fig. 6o). Disruption of the alveolar epithelial lining
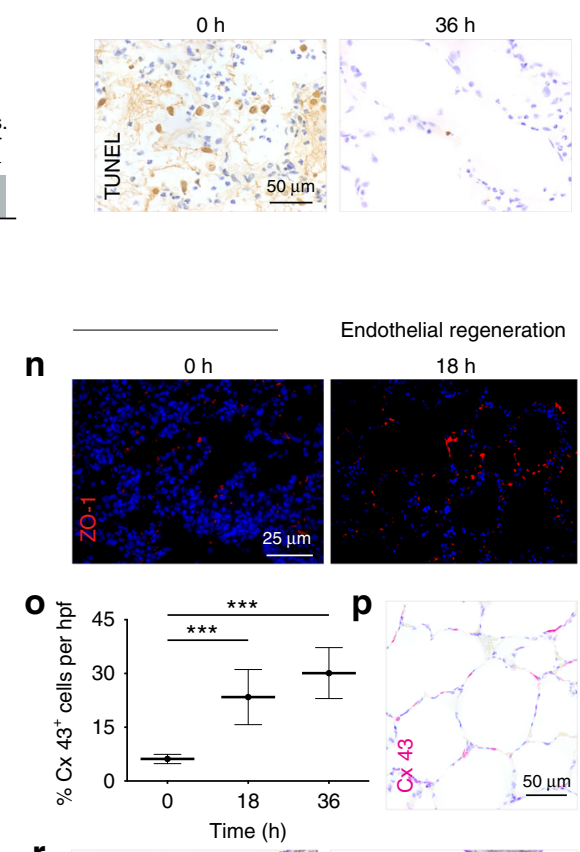

$\mathbf{r} 0 \mathrm{~h}$

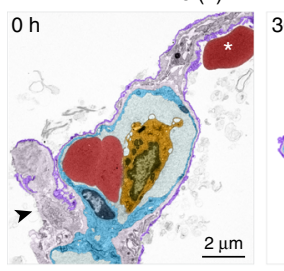

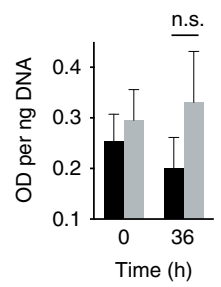

$36 \mathrm{~h}$

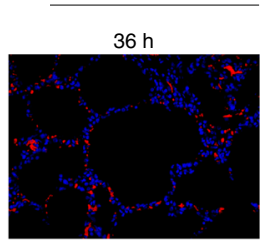

q

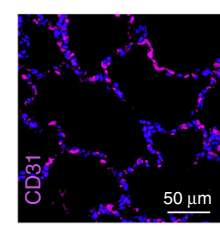

$\mathbf{S}$

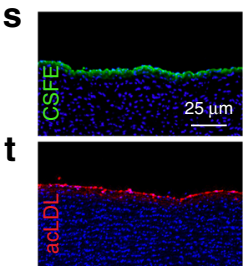

and the presence of inflammatory cells, extravascular erythrocytes, and extracellular debris confirmed lung injury and a compromised blood-gas barrier (Fig. 6r; Supplementary Fig. 7b). After $36 \mathrm{~h}$ of cross-circulation, the pulmonary microvasculature of injured lungs appeared intact by immunostaining for CD31 (Fig. 6q), and the transmission electron micrographs (Fig. 6r; Supplementary Fig. 7b) resembled those of control lungs (Supplementary Fig. 7c), with clear restoration of the alveolar epithelial lining and intraseptal capillary geometry and architecture (Supplementary Fig. 7b). Viability of the pulmonary 
Fig. 6 Cellular recovery and regeneration of injured lungs. Histologic analysis of airway and vascular recovery. a Large airway: Alcian blue $0 \mathrm{~h}$ with marginating PMNs (arrows) and $36 \mathrm{~h}$ with goblet cells (arrow), periodic acid-Schiff (PAS) stain demonstrating loss of epithelial integrity and airway cilia at $\mathrm{O} \mathrm{h}$ and restoration of ciliated epithelial lining at $36 \mathrm{~h}$ (arrows). b Distal airway: Alcian blue and PAS stain $\mathrm{O}$ h with alveolar edema (star), pentachrome $\mathrm{O} \mathrm{h}$ with alveolar infiltrates (arrow). c Vascular network: Alcian blue $\mathrm{O}$ h with marginating PMNs (arrows), PAS stain $\mathrm{O}$ h with perivascular edema (star), pentachrome $\mathrm{O} \mathrm{h}$ with intimal thickening (star). d Neutrophil quantification. e Myeloperoxidase (MPO) activity. f Macrophage quantification by CD163 immunostaining. Student's t-tests, ${ }^{\star \star \star} p<0.001,{ }^{\star \star} p<0.01$, or $p>0.05$, not significant (n.s.). $\mathbf{g}$ TUNEL staining of injured lungs at $0 \mathrm{~h}$ and $36 \mathrm{~h}$. $\mathbf{h}$ Metabolic activity of injured and control lungs at $\mathrm{O}$ h and $36 \mathrm{~h}$, Student's $t$-test for Injured versus Control, not significant (n.s.). i Aquaporin 5 (Aqp 5 ) and (j) ZO-3 immunostaining of injured lungs at 0,18 , and $36 \mathrm{~h}$. Recovery of surfactant in type Il cells: (k) Loss of surfactant protein C (SPC) at 0 h, with recovery at 36 h. I Transmission electron micrograph (TEM) of type II cell with loss of lamellar bodies containing surfactant (white arrow) at $0 \mathrm{~h}$, and TEM of type II cell with lamellar bodies containing surfactant (white arrow) with active surfactant secretion (black arrow) at $36 \mathrm{~h}$. $\mathbf{m}$ Function of type II pneumocytes demonstrated by uptake of fluorescently labeled surfactant protein B (BODIPY-SBP) at $36 \mathrm{~h}$. $\mathbf{n}$ ZO-1 immunostaining at $0,18,36 \mathrm{~h}$. o Quantification of connexin $43^{+}$cells at $0,18,36 \mathrm{~h}$ (Student's t-tests, ${ }^{\star \star \star} p<0.001$ ), with $\mathbf{p}$ connexin 43 immunostaining at $36 \mathrm{~h}$. q CD31 immunostaining at $36 \mathrm{~h}$. $\mathbf{r}$ TEM (pseudocolored) of pulmonary capillaries at $\mathrm{O}$ h showing an extravascular red blood cell (red) indicating alveolar hemorrhage (star), disruption of type I cell membrane (purple, arrow), and intra-capillary leukocyte (orange) adjacent to microvascular endothelial cell (blue), and $36 \mathrm{~h}$ demonstrating intact blood-gas barrier. Vascular viability and function at $36 \mathrm{~h}$ : uptake of $\mathbf{s}$ CSFE and $\mathbf{t}$ acetylated low-density lipoprotein (acLDL). All graphs represent data from $n=8$ lungs. All values represent mean \pm standard deviation

endothelium after $36 \mathrm{~h}$ of cross-circulation was confirmed by uptake of carboxyfluorescein succinimidyl ester (CFSE; Fig. 6s) and acetylated low-density lipoprotein (acLDL; Fig. 6t).

\section{Discussion}

We describe the application of interventional cross-circulation-a strategy for prolonged, systemically regulated normothermic extracorporeal organ support-to regenerate severely damaged lungs (Supplementary Movie 1). Our results suggest that lungs injured by gastric aspiration, a common lung injury rendering donor lungs unsuitable for transplantation: (i) can be maintained on cross-circulation support without decline in lung function or tissue integrity, (ii) are amenable to repeated therapeutic interventions, and (iii) display evidence of cellular regeneration and improved function over the course of $36 \mathrm{~h}$.

Aspiration of gastric contents was used as an injury model due to its: (i) reproducibility and established protocols ${ }^{21,36}$, (ii) severity of functional decline and tissue derangement, and (iii) clinical relevance in the context of acute lung injury and lung transplantation. Lung injury occurs in two distinct phases ${ }^{37}$. In the first phase (0-2 h after aspiration), damage results in the disruption of alveolar type I cell membranes and increased microvascular permeability. Alterations in the blood-gas barrier lead to extravasation of fluid and protein into the airways and alveoli, leading to pulmonary edema, decreased compliance, and progressive hypoxia ${ }^{38}$. In the second phase $(2-6 \mathrm{~h}$ after aspiration), an inflammatory cascade results in neutrophil migration and the release of inflammatory cytokines such as IL-1 $\beta$ and TNFa by activated alveolar macrophages ${ }^{39,40}$. These processes in conjunction with the release of reactive oxygen species, free radicals, and proteases by infiltrating immune cells ${ }^{41,42}$ exacerbate lung injury.

Normothermic cross-circulation allowed for $36 \mathrm{~h}$ of repeated therapeutic interventions (Fig. 1b) that aided in the recovery of damaged lungs. Airway lavage was effective in ameliorating the caustic effects of gastric aspiration by removing particulate and cellular debris ${ }^{43}$ and by neutralizing $\mathrm{pH}$, thereby decreasing proteolytic enzyme concentration and activity (Fig. 3, a-h). While the delivery of exogenous surfactants effectively replenishes depleted and degraded endogenous surfactants and improves compliance ${ }^{44}$, beneficial effects only occur after inhibitory plasma proteins and fluid are removed by lavage ${ }^{45}$. Accordingly, in our therapeutic intervention regimen, surfactant replacement was performed after bronchoalveolar lavage. Alveolar recruitment maneuvers improve ventilation-perfusion matching and arterial oxygenation ${ }^{46}$, and have been shown to improve clinical outcomes ${ }^{47}$. Administration of surfactant enhances the spatial distribution of lung ventilation via recruitment, which recovers gas exchange ${ }^{48}$ through restoration of ventilation-perfusion match.

Pepsin, a biomarker of gastric aspiration ${ }^{49,50}$, has recently been linked to allograft injury and bronchopulmonary dysplasia in transplant patients ${ }^{51}$. Pepsin-induced cytotoxicity and release of IL- 6 and IL- 8 peaks at $\mathrm{pH} 1.5-2.5^{52}$. After $12 \mathrm{~h}$ of crosscirculation and repeated bronchoalveolar lavage, pepsin concentrations in BAL fluid significantly decreased, $\mathrm{pH}$ of BAL fluid normalized to $\mathrm{pH}>5$, and IL-6 and IL-8 concentrations decreased significantly (Fig. 3, Supplementary Fig. 4). Elevated levels of IL-8 in BAL fluid perpetuate aspiration injury via IL-8 dependent mechanisms ${ }^{53,54}$. Notably, initially high levels of IL-8 in injured lungs normalized to control levels after $12 \mathrm{~h}$ of interventional cross-circulation. Similarly, levels of other key inflammatory cytokines (e.g., IFN $\gamma$, IL- $1 \beta$, and TNF $\alpha$ ) decreased significantly in BAL fluid from injured lungs over the course of $36 \mathrm{~h}$ of interventional cross-circulation (Fig. 3h, Supplementary Fig. 4c, Supplementary Table 9). Gastric aspiration also increases susceptibility to respiratory pathogens and bacterial infections ${ }^{55,56}$. Microbial cultures of BAL fluids from injured and control lungs contained normal respiratory flora and were negative for pathologic growth, demonstrating that repeated interventions in extracorporeal lungs can be performed without introducing pathogens.

Over $36 \mathrm{~h}$ of cross-circulation, injured lungs showed recovery of mechanical compliance to $68 \%$ of the average compliance of control lungs-a more than six-fold improvement. Significant changes in pressure-volume (PV) loops were consistent with this degree of recovery and did not resemble stereotypical PV loop hysteresis until after $24 \mathrm{~h}$ of cross-circulation (Fig. $4 \mathrm{a}, \mathrm{b}$ ). The initial average $\mathrm{PaO}_{2} / \mathrm{FiO}_{2}$ of injured lungs was $<90 \mathrm{mmHg}(11 \%$ of the average $\mathrm{PaO}_{2} / \mathrm{FiO}_{2}$ in control lungs) and increased to $>640$ $\mathrm{mmHg}$ ( $83 \%$ of the average $\mathrm{PaO}_{2} / \mathrm{FiO}_{2}$ in control lungs). Notably, following $36 \mathrm{~h}$ of cross-circulation there was no significant difference between the $\mathrm{PaO}_{2} / \mathrm{FiO}_{2}$ of injured and control lungs (Fig. 4c).

Lung injury scores consistently decreased across all categories, with the greatest reductions in alveolar PMN cells, edema, and apoptosis (Fig. 5l, Supplementary Fig. 5g-i; Supplementary Fig. 6). Notably, we demonstrated: (i) longitudinal expression and regeneration of cell surface markers of the pulmonary epithelium and endothelium, and tight and gap junction proteins, (ii) recovery of functional subcellular structures (e.g., intracellular vesicles), and (iii) cellular function and integrity throughout $36 \mathrm{~h}$ of extracorporeal support (Fig. 6).

Acute lung injury affects the alveolar epithelium and microvascular endothelium, and specifically reduces the expression of 
aquaporin 5, which plays an essential role in water transport ${ }^{57}$. Following aspiration, aquaporin 5 was drastically reduced in injured lungs. After $36 \mathrm{~h}$ of interventional cross-circulation, aquaporin 5 expression was similar in injured and control lungs (Fig. 6i, Supplementary Fig. 7a). Additionally, epithelial tight junction protein $\mathrm{ZO}-3$ and microvascular tight junction protein ZO-1 displayed similar expression patterns of recovery, with very low initial levels and significantly higher levels reaching those in control lungs after $36 \mathrm{~h}$ of cross-circulation. These data, along with decreased lung weight (Supplementary Fig. 5e), improved gas exchange and compliance (Fig. 4), and decreased alveolar edema observed by light and electron microscopy (Figs. 5, 6), strongly suggest that interventional cross-circulation enabled significant cellular regeneration in the airway and vascular compartments, resulting in functional restoration of the alveolarcapillary membrane in extracorporeal lungs.

Decrease in surfactant protein $\mathrm{C}$, a marker of type II pneumocytes ${ }^{58}$, in distal regions of injured lungs resulted in the loss of surfactant-filled lamellar bodies in type II cells. After $36 \mathrm{~h}$ of interventional cross-circulation, significant increases in SPC expression and restoration of intracellular surfactant-filled lamellar bodies were observed (Fig. 6k, 1). Airway regeneration was demonstrated by the restoration of: (i) contiguous type I cell membranes, (ii) a ciliated epithelial brush border, and (iii) subcellular structures including mucus-filled vesicles in airway goblet cells and surfactant-filled lamellar bodies in alveolar type II cells. These data suggest extensive epithelial cellular regeneration in the airway that has not been demonstrated by other lung support systems (Supplementary Table 1-3). In addition, increased expression of connexin 43 (Fig. 6o, p) correlated with the recovery of severely damaged lungs over $36 \mathrm{~h}$ of support, during which the injured pulmonary vascular network not only remained viable but also substantially regenerated. We hypothesize that the degree of cellular regeneration achieved is the result of the beneficial effects of therapeutic interventions, physiologic homeostatic maintenance and prolonged support provided by crosscirculation with a living host.

The present study has several limitations: (1) While the use of inbred swine enabled recovery and regeneration of injured lungs with minimal donor-recipient immunologic cross-reactivity, future studies will need to assess the effects of immunosuppression (necessary for lung transplantation) on lung recovery. (2) This study assessed recovery from lung injury $6 \mathrm{~h}$ after gastric aspiration, a period within which a severe inflammatory response develops. Clinically, aspiration events often precede interventional recovery by more than $6 \mathrm{~h}$, and additional studies are needed to extend this approach to lungs enduring a longer exposure to aspiration pneumonitis. Nevertheless, the injury model and therapeutic regimen used in this study may also represent an opportunity to inform clinical practice in cases of known or suspected gastric aspiration (e.g., in trauma patients, patients found down prior to admission to the hospital, or patients intubated in the field by first responders). (3) To maintain appropriate controls, only a single lung was injured in each experiment, while in the clinical setting, patients frequently develop bilateral aspiration pneumonitis. (4) According to multiple assessment criteria, including radiography, pressure-volume loops, compliance, histologic analyses, lung injury scores, and cell-specific functional markers such as aquaporin 5 and surfactant protein $\mathrm{C}$, injured lungs improved to reach transplantation criteria, but did not achieve full recovery. Future studies should investigate the functional capacity of lungs following transplantation, and if longer support and recovery times would be of greater benefit.

Beyond demonstrating extracorporeal regeneration of severely damaged lungs, this study resulted in the development of technical methodologies. During ex vivo organ perfusion, advanced thermography ${ }^{59}$ can be used for the identification of flow and circuit failures ${ }^{60}$, and enable real-time visualization of rewarming during lung reperfusion ${ }^{24}$. We extended thermography to assessing global surface temperatures and conducting localized thermal recovery tests as a diagnostic for non-invasive evaluations of injured extracorporeal lungs. We also found that exosome concentrations in BAL fluids from injured lungs were elevated and increased over the first $12 \mathrm{~h}$ of cross-circulation. As injured lungs recovered, exosome concentrations decreased and approached levels in control lungs (Fig. 3g). Profiling of exosome surface markers and cargo was beyond the scope of this study but represents an opportunity for future investigation, as exosomes in BAL fluid may represent a biomarker to diagnose, grade, and monitor the trajectory of lung injury and recovery.

Envisioned clinical applications of an interventional crosscirculation platform are motivated by the thousands of patients who die every year awaiting suitable organs for transplantation. The recovery of an unsuitable organ may represent their only chance for transplantation and survival. We envision that prolonged extracorporeal support by interventional cross-circulation could be used to recover and regenerate severely damaged organs (Supplementary Fig. 9). Furthermore, as humanized swine and immunosuppressive regimens are being developed for applications in xenotransplantation, the utility of a non-human host should also be assessed in a xenogeneic cross-circulation system for the recovery of human donor organs unacceptable for transplantation.

In summary, we report: (i) the development of an interventional extracorporeal organ support platform capable of systemic regulation, (ii) the use of non-invasive diagnostics (dynamic surface thermography, exosomes), and (iii) evidence of cellular regeneration of severely damaged extracorporeal lungs. Furthermore, we demonstrate: longitudinal expression and regeneration of cell markers of the pulmonary epithelium and endothelium, recovery of functional subcellular structures, and cellular function and integrity throughout $36 \mathrm{~h}$ of interventional cross-circulation. Our results also establish a benchmark for molecular and cellular analytics of extracorporeal lung regeneration (Supplementary Table 4). We envision that interventional cross-circulation may be used to investigate regeneration of other damaged organs (e.g., hearts, kidneys, livers), where expansion of donor pools through the salvage of severely damaged organs could lead to more organ transplantations.

\section{Methods}

Animals. Sixteen miniature swine (eight donor-recipient pairs) from a herd of partially inbred swine were utilized in this study. Pairs with well-defined major histocompatibility complex (MHC) loci were selected. Immunogenetic characteristics and selective breeding methods have been previously reported ${ }^{61}$. Animals were 5-8 months of age, with a median weight of $39.2 \mathrm{~kg}$ (range: $30.6-53.5 \mathrm{~kg}$ ). All studies complied with the relevant ethical regulations for animal testing and research. Approval for this study was received from the Institutional Animal Care and Use Committee at Columbia University. Animal care and procedures were conducted in accordance with the US National Research Council of the National Academies Guide for the Care and Use of Laboratory Animals, 8th Edition.

Standardization of gastric contents for gastric aspiration injury model. Gastric contents were collected from six swine (of the same herd) deprived of food for $12 \mathrm{~h}$ and standardized according to methods previously described $21,25,36$. Briefly, animals $(n=6)$ were sedated, and an orogastric tube ( $36 \mathrm{Fr})$ was inserted and engaged to suction. Gastric contents collected from swine were admixed, strained through sterile surgical gauze to remove large particulates, normalized with sterile hydrochloric acid to a standard $\mathrm{pH}$ of $2.00 \pm 0.05$, aliquotted, stored at $-80^{\circ} \mathrm{C}$, and thawed immediately prior to experimental use (Supplementary Fig. 1a). The concentration of total non-sulfated bile acids $(416 \pm 208 \mu \mathrm{M})$ and presence of pepsin in standardized gastric contents were measured and confirmed using a Bile Acid Assay Kit (Sigma-Aldrich) Pepsin ELISA kit (LSBio) according to the manufacturer's instructions. Microbial cultures of gastric contents were negative for all aliquots. 
In vivo lung injury. Donor swine $(n=8)$ underwent general anesthesia via intramuscular induction with Telazol $\left(5 \mathrm{mg} \mathrm{kg}^{-1}\right.$, Zoetis) and buprenorphine hydrochloride $\left(0.03 \mathrm{mg} \mathrm{kg}^{-1}\right.$, Hospira). Following induction and intubation, the anesthetic regimen consisted of continuous infusions of midazolam $\left(1.5 \mathrm{mg} \mathrm{kg}^{-1} \mathrm{~h}^{-1}\right.$, Akorn), fentanyl citrate $\left(0.1 \mathrm{mg} \mathrm{kg}^{-1} \mathrm{~h}^{-1}\right.$, West-Ward), and inhaled isoflurane (1-5\% in oxygen, Henry Schein). Gastric aspiration injury was adapted from previously established injury models ${ }^{21,25,36}$. Following donor sedation, a bronchoscope was introduced into the lower lobe of a single lung, and the location of the bronchoscope tip was confirmed by chest X-ray. Standardized gastric contents $\left(2 \mathrm{~mL} \mathrm{~kg}^{-1} ; \mathrm{pH} 2\right)$ were delivered bronchoscopically while withdrawing the tip of the bronchoscope to a position approximately $1 \mathrm{~cm}$ distal to the carina to avoid introduction of gastric contents into the contralateral (control) lung. This distance was established prior to delivery of standardized gastric contents by measuring the length of the bronchoscope as it was advanced past the carina. After delivery, animals remained under general anesthesia and standardized gastric contents remained in donor lungs for $6 \mathrm{~h}$ to allow development of a unilateral acute lung injury in vivo.

Validation of gastric aspiration injury. Following unilateral bronchoscopic delivery of standardized gastric contents into donor lungs, donor vitals and ventilator settings were continuously recorded. Chest X-rays, airway bronchoscopic images, and hemogases were obtained at baseline and every $2 \mathrm{~h}$ for $6 \mathrm{~h}$. Donor blood samples were collected at baseline and $6 \mathrm{~h}$ after gastric aspiration to assess serum levels of inflammatory markers (GM-CSF, IFN $\gamma$, IL-1 $\alpha$, IL-1 $\beta$, IL-1 r $\alpha$, IL-2, IL-4, IL-6, IL-8, IL-10, IL-12, IL-18, P-Selectin, and TNFa) by multiplex array analysis (Eve Technologies) and hemolytic markers (D-dimer, fibrinogen, and plasma free hemoglobin) by commercially available enzyme-linked immunosorbant assays (ELISAs). A list of the ELISA kits used is provided in Supplementary Table 11. BAL fluid samples were collected from injured and control lungs at baseline and $6 \mathrm{~h}$ after gastric aspiration to assess the airway response to gastric aspiration. Inflammatory markers (GM-CSF, IFN $\gamma$, IL-1 $\alpha$, IL-1 $\beta$, IL-1r $\alpha$, IL-2, IL-4 IL-6, IL-8, IL-10, IL-12, IL-18, and TNF $\alpha$ ) in BAL fluid samples were quantified by multiplex array analysis (Eve Technologies), M30 by commercially available ELISA, and cellular contents in BAL fluid were analyzed by Kwik-Diff staining or periodic acid-Schiff staining. Gross images of injured lungs were obtained prior to explant using an interchangeable lens digital camera (Sony).

Histopathologic analyses of in vivo lung injury. Tissue samples were collected from lung segments (lower lobes and posterior segments of upper lobes) of injured and control lungs according to a pre-determined lung map (Supplementary Fig. 4a) to avoid sampling bias and enable randomized sampling of injured and control lungs. A surgical stapler with medium/thick reloads was used to obtain lung samples. Specimens were immediately fixed in cold phosphate-buffered $4 \%$ paraformaldehyde for $48-72 \mathrm{~h}$, embedded in paraffin, and sectioned at $3 \mu \mathrm{m}$ or $5 \mu \mathrm{m}$ thickness. All sections were stained with hematoxylin and eosin (H\&E) and pentachrome and examined via light microscopy. Immunohistochemical staining was conducted for neutrophil elastase (airway and alveolar polymorphonuclear cells) and caspase 3 (early apoptotic cells). Late apoptosis was detected on tissue sections using a TdT-mediated dUTP nick end-labelling (TUNEL) assay. Pathologic review was performed by a pulmonary pathologist blinded to the samples and study protocol. All slides (H\&E, immunohistochemical staining for neutrophil elastase and caspase 3, and TUNEL) were randomized, arbitrarily numbered, and delivered to the pathologist without reference to experimental time points or conditions $s^{55}$. To evaluate gastric aspiration injury, a modified lung injury score was developed from previously described methods ${ }^{56,57}$. Scoring (Supplementary Table 6) was based on airway polymorphonuclear cells per high-power field (hpf) $\left(\times 40 ; 0.2 \mathrm{~mm}^{2} ; 0 \Rightarrow 0,1 \Rightarrow 1-25,2 \Rightarrow 26-50,3 \Rightarrow>50\right)$, alveolar polymorphonuclear cells per hpf $\left(\times 40 ; 0.2 \mathrm{~mm}^{2} ; 0 \Rightarrow 0,1 \Rightarrow 1-25,2 \Rightarrow 26-50,3 \Rightarrow>50\right)$, alveolar edema $(0 \Rightarrow<5 \%$ of all alveoli contained edema fluid, $1 \Rightarrow 6-25 \%, 2 \Rightarrow 26-50 \%$, $3 \Rightarrow>50 \%$ ), interstitial infiltrate (lymphocytes and neutrophils in the interstitium around vessels and airways and in alveolar septa and pleura; $0 \Rightarrow$ none per hpf, $1 \Rightarrow<50$ per hpf, $2 \Rightarrow 50-100$ per hpf, $3 \Rightarrow>100$ per hpf), interstitial edema (perivascular and peribronchial spaced expanded with edematous fluid; $0 \Rightarrow$ none per hpf, $1 \Rightarrow 1 \mathrm{x}$ width vessel media, $2 \Rightarrow \geq 2 \mathrm{x}$ width vessel media), and total number of apoptotic cells per hpf $\left(\times 40 ; 0.2 \mathrm{~mm}^{2} ; 0 \Rightarrow<2,1 \Rightarrow 3-5,2 \Rightarrow 6-10,3 \Rightarrow>10\right)$. Numbers for early and late apoptosis, as quantified by caspase 3 and TUNEL, were combined into a single apoptosis score. Lung injury scores of injured and control lungs were compared at $6 \mathrm{~h}$ after gastric aspiration as an index of lung injury following unilateral delivery of standardized gastric contents.

Procurement of injured lungs. Prior to skin incision, animals were prepped and draped in standard sterile fashion and antibiotics were given (Cefazolin, $30 \mathrm{mg} \mathrm{kg}^{-1}$. WG Critical Care). Following median sternotomy, a heparin bolus (30,000 U) was administered intravenously (Sagent Pharmaceuticals), and the main pulmonary artery (PA) was cannulated. Autologous blood was collected via the PA cannula. Blood was stored in citrate-phosphate-dextrose collection bags (Chinook Medical) at $8{ }^{\circ} \mathrm{C}$. Following exsanguination and presence of a non-perfusing cardiac rhythm, a cold anterograde low-potassium dextran flush (Perfadex, Vitrolife) with alprostadil (25 mg kg-1 , Prostin VR Pediatric, Pfizer) was delivered via the main PA cannula, and the inferior vena cava and left atrial appendage were transected. Concurrently with the flush, topical cooling with sterile ice was applied. Once the flush was complete additional dissection was completed, and the lungs were inflated to a sustained airway pressure of $15 \mathrm{cmH}_{2} \mathrm{O}$. With the lungs inflated and all lobes recruited, the trachea was stapled (Endo GIA, Medtronic), and the heart and lungs were explanted en-bloc. Working on a sterile back table, the heart was removed. Cannulation with the bio-bridge was facilitated by leaving a circumferential cuff (height of 3-5 mm) of the left atrium. Next, a retrograde flush with cold Perfadex $\left(20 \mathrm{~mL} \mathrm{~kg}^{-1}\right)$ was performed. To prepare the bio-bridge, the aortic arch was first dissected, and the left subclavian and brachiocephalic branches were stapled. A sufficient length of aorta was left on either side of these vessels to facilitate placement of the pulmonary vein cannula into one end and attachment to the left atrial cuff at the other (Supplementary Fig. 2a-f).

Lung cannulation. To enable inflow via the pulmonary artery, an 18-20 Fr cannula was secured using a purse-string $5-0$ prolene suture, 0 silk ties, and TourniKwik tourniquet (Medtronic). To enable ventilation, a $7.5-8.0 \mathrm{~mm}$ cuffed endotracheal tube (Sheridan) was placed within the trachea and secured with 0 silk ties.

Single lung venous cannulation. The aortic arch, serving as an endothelialized biological bridge (bio-bridge) between the lung and the extracorporeal circuit, was connected to the left atrial cuff with a running 6-0 prolene suture (Ethicon). Outflow through the bio-bridge was facilitated by the placement of a 36 Fr crenellated drainage cannula. This venous drainage cannula was secured in place with 2-0 Ti-Cron ties (Covidien) or 0 silk ties. To assess unilateral gas exchange function of injured or control lungs, two $20 \mathrm{G} \times 12 \mathrm{~cm}$ cannulas (Arrow International) (Supplementary Fig. 2e) were placed through the wall of the bio-bridge (aortic arch) and inserted into the left and right pulmonary veins. The position of each single lung venous cannula was confirmed by X-ray (Fig. 1d; Supplementary Fig. $2 \mathrm{~g}$ ). Intravenous tubing (diameter: $3 \mathrm{~mm}$ ) was connected to each single lung venous cannula, led out of the organ preservation chamber, and connected to a venous blood port (Supplementary Fig. $2 \mathrm{~h}$ ) to enable single lung venous blood hemogas analysis.

Once cannulated, lungs were flushed with cold normal saline to remove air and prime the cannulas. Within the preservation chamber (XVIVO), a top-loading balance (Denver Instrument Company) was placed, followed by a double-lined organ basin with warm recirculating saline (Supplementary Fig. 3). Lungs were positioned in the prone position within the basin, and the PA, PV, and endotracheal tube were secured. Whole blood collected during donor lung procurement was used to prime the circuit. Next, the circuit was de-aired, and connected to extracorporeal lungs. Initial flow rates were set to $5-10 \%$ of the estimated cardiac output, with a target pulmonary artery pressure of $<15 \mathrm{mmHg}$, and pulmonary vein pressure of $3-5 \mathrm{mmHg}$.

Cross-circulation. Recipient swine $(n=8)$ underwent sedation and general anesthesia in the same fashion as donor swine. All recipients underwent endotracheal intubation and were continuously ventilated throughout the duration of the study. Prior to skin incision, antibiotics (Cefazolin; $30 \mathrm{mg} \mathrm{kg}^{-1}$ ) was given and re-dosed every $8 \mathrm{~h}$. An auricular arterial line (Arrow International) was placed for hemodynamic monitoring and periodic blood sampling. Femoral arterial lines were used if auricular placement was unsuccessful. Exposure of the left and right internal jugular veins was accomplished via bilateral neck cut-downs. Cannulation with 16-18 Fr catheters was performed in standard fashion using the Seldinger technique. A heparin bolus $(15,000 \mathrm{U})$ was administered just prior to cannulation. Immediately prior to initiation of cross-circulation, $1 \mathrm{~g}$ methylprednisolone (APP Pharmaceuticals) and $500 \mathrm{mg}$ calcium chloride (Hospira) were intravenously administered to recipient swine. Donor blood was used to prime the circuit, and tubing was spliced to connect the recipient swine to the extracorporeal circuit, marking the start of cross-circulation (Supplementary Fig. 3a, b).

Ventilation was initiated within the first $10 \mathrm{~min}$ of cross-circulation while the circuit and lungs were allowed to acclimate to ambient temperature. Initial settings were as follows: volume control mode, respiratory rate $6-8 \mathrm{bpm}$, tidal volume (TV) 6-8 $\mathrm{mL} \mathrm{kg}^{-1}$, positive end-expiratory pressure (PEEP) $5 \mathrm{cmH}_{2} \mathrm{O}$, and $\mathrm{FiO}_{2} 40 \%$ (Oxylog 3000 plus; Dräger). To recruit atelectatic lung regions, ventilatory maneuvers included increasing PEEP (up to $10 \mathrm{cmH}_{2} \mathrm{O}$ ), TV, and by performing inspiratory hold maneuvers (up to $25 \mathrm{cmH}_{2} \mathrm{O}$ ). Manual recruitment maneuvers were used if ventilation strategies failed. Thermal images of the lung were captured at periodic time points throughout the duration of each procedure using a FLIR T430sc infrared camera.

Circuit elements consisted of the following: main console (Jostra HL-20 pump console; Maquet), disposable pump (Rotaflow centrifugal pump; Maquet), softshell reservoir (Maquet), and three-eighths inch tubing (Smart coated tubing; LivaNova). Continuous monitoring and recording (VIPER clinical interfacing software; G2 v1.26.4; Spectrum Medical) of pressures (pulmonary artery and vein), flows (pulmonary artery and vein), and temperatures were performed. The recipient was maintained on a continuous heparin infusion throughout the duration of cross-circulation (initial rate of $25 \mathrm{U} \mathrm{kg}^{-1} \mathrm{~h}^{-1}$ ), and activated clotting time (ACT) was measured using a whole blood micro-coagulation system (Hemochron; Accriva Diagnostics). Adjustments to the heparin drip were made in order to maintain a target ACT value of 250-350 s. Physiological parameters of the 
recipient were continuously monitored and recorded using a multi-parameter Advisor vital signs monitor (SurgiVet). These included: heart rate, electrocardiogram, blood pressure (cuff and arterial line pressure), mean arterial pressure (MAP), oxygen saturation $\left(\mathrm{SpO}_{2}\right)$, end-tidal $\mathrm{CO}_{2}$, temperature, and respiratory rate. Analysis of recipient vitals, hemogas, biochemistry, hemolysis, electrolytes, and serum cytokines throughout $36 \mathrm{~h}$ of cross-circulation is provided in Supplementary Table 7, 8 .

Therapeutic interventions. Recovery of injured lung function during crosscirculation was facilitated by a series of interventions within the scope of standard clinical practice. A standardized 30-min interventional procedure (Fig. 1b) was performed on injured lungs at $0,2,4,6,12$, and $18 \mathrm{~h}$ of cross-circulation: three cycles of bronchoalveolar lavage $(15 \mathrm{~min})$, surfactant replacement $(5 \mathrm{~min})$, and alveolar recruitment $(10 \mathrm{~min})$, followed by functional assessments of injured and control lungs by single lung analysis of hemogases and dynamic compliance. All airway bronchoscopy, bronchoalveolar lavage, and surfactant delivery were performed with a $3.8 \mathrm{~mm}$ flexible bronchoscope (aScope 3, Ambu).

Bronchoalveolar lavage. At $0,2,4,6,12$, and $18 \mathrm{~h}$ of cross-circulation, bronch oalveolar lavage was used to clear gastric contents, secretions, and cellular debris from injured lungs. Sterile normal saline $\left(5 \mathrm{~mL}\right.$ segment $\left.^{-1}\right)$ was bronchoscopically injected into each injured lung segment and immediately aspirated. To therapeutically intervene in an injured porcine lung (e.g., the left porcine lung consists of two lobes (cranial and caudal) with a total of ten bronchopulmonary segments ${ }^{62}$ ) the volume of lavage fluid per therapeutic intervention was $\sim 50 \mathrm{~mL}$. Fiveminute intervals were interspersed throughout the lavage procedure to minimize interruptions in lung ventilation. Accordingly, similar to previously described protocols $^{11}$, the total volume of lavage fluid was $\sim 300 \mathrm{~mL}$.

Surfactant replacement. Immediately following bronchoalveolar lavage of injured lungs at $0,2,4,6,12$, and $18 \mathrm{~h}$ of cross-circulation, exogenous surfactant (bovine lipid extract surfactant, BLES; Biochemicals) was delivered bronchoscopically into injured lungs. Bovine lipid extract surfactant was stored at $-20^{\circ} \mathrm{C}$ and warmed to room temperature immediately prior to administration (Supplementary Fig. 8g). During each therapeutic intervention, one vial of bovine lipid extract surfactant ( $3 \mathrm{~mL} ; 27 \mathrm{mg}$ phospholipids $\mathrm{mL}^{-1}$ ) was administered and dispersed throughout injured lungs. Accordingly, injured lungs received a total dose of bovine lipid extract surfactant of $12.5 \mathrm{mg}$ phospholipid $\mathrm{kg}^{-1}$ (donor swine body weight), consistent with surfactant replacement strategies previously described ${ }^{11}$.

Alveolar recruitment. Following bronchoalveolar lavage and surfactant replacement, alveolar recruitment maneuvers were performed to transiently increase the airway positive pressure gradient, resulting in the opening of non-aerating lung units and increased vital capacity, while avoiding barotrauma and the deleterious effects of hyperinflation. Ventilation strategies included increasing tidal volume by $15-20 \%$, increasing positive end-expiratory pressure (PEEP) up to $12.5 \mathrm{cmH}_{2} \mathrm{O}$, and performing inspiratory hold maneuvers (sustained inflation) up to $25 \mathrm{cmH}_{2} \mathrm{O}$. When ventilation strategies were insufficient, manual recruitment maneuvers were performed by an experienced lung transplant surgeon in a manner consistent with standard practices used during clinical lung transplantation. During inspiration, gentle manual compression was applied to areas of aerated lung, thereby directing ventilation and recruitment toward areas of non-aerating atelectatic lung. Peak inspiratory pressures were monitored and maintained below $30 \mathrm{cmH}_{2} \mathrm{O}$. Accordingly, during each therapeutic intervention, injured lungs were subjected to $10 \mathrm{~min}$ of alveolar recruitment maneuvers prior to returning all ventilator settings to a lung protective ventilation strategy. Injured lungs underwent a total alveolar recruitment time of $\sim 60 \mathrm{~min}$.

Recipient monitoring and blood analysis. Hourly blood samples were drawn from an auricular arterial line (or femoral arterial line if auricular placement was unsuccessful), and analyzed using an epoc point-of-care blood analysis system (Epocal). Additional samples were collected in test-specific specimen vials (BD Vacutainer) every $4 \mathrm{~h}$ to obtain the following: complete blood count, liver function tests, basic metabolic panel, lactate dehydrogenase, and coagulation panels (Antech Diagnostics). Additional hemolytic markers (D-dimer, plasma free hemoglobin fibrinogen,) and inflammatory markers (GM-CSF, IFN $\gamma$, IL- $1 \alpha$, IL-1 $\beta$, IL-1r $\alpha$, IL-2, IL-4, IL-6, IL-8, IL-10, IL-12, IL-18, P-selectin, and TNF- $\alpha$ ) were analyzed in triplicate by the Discovery Assay Pig Cytokine Array (Eve Technologies) or by commercially available enzyme-linked immunosorbant assays (ELISAs) according to the manufacturer's instructions. A list of the ELISA kits used is provided in Supplementary Table 11 .

Extracorporeal lung monitoring and functional analyses. Blood samples were drawn from the main pulmonary artery cannula (blood entering both lungs), main pulmonary vein cannula (blood exiting both lungs), and single lung venous cannulas (blood exiting each lung) every $6 \mathrm{~h}$ and analyzed in the same fashion as recipient blood samples. Temporary changes in ventilation settings (minute ventilation and $\mathrm{FiO}_{2}$ ) were made according to a lung performance challenge protocol in order to further assess extracorporeal lung performance every $6 \mathrm{~h}$. Immediately following the challenge, a subsequent set of blood samples were drawn from the pulmonary artery and pulmonary vein cannulas. Dynamic compliance $\left(\mathrm{C}_{\mathrm{dyn}}=\mathrm{TV} /\right.$ (PIP - PEEP); $\mathrm{mL} \mathrm{cmH}_{2} \mathrm{O}^{-1}$ ) of extracorporeal lungs was calculated every $6 \mathrm{~h}$. Pressure and volume recordings of lungs were measured using a custom measurement system consisting of sensors for pressure and airflow and a data acquisition device (Arduino Uno). Data collected with this system were processed, and pressure-volume loops were generated using MATLAB v. R2016b (Mathworks). Lung weight was obtained every $6 \mathrm{~h}$ using the top-loading scale (Denver Instrument Company) within the organ chamber. To ensure accurate recordings, the basin and contents were tared (zeroed) at each time point. X-ray images of lungs were obtained using a PXP-16HF portable X-ray unit (United Radiology Systems) at $2.2 \mathrm{mAs}$ and $90 \mathrm{kVp}$.

Single lung ventilation. To enable single lung ventilation and single lung compliance measurements, an endobronchial blocker (7 Fr; Cook Medical) (Supplementary Fig. 2i-k) was inserted into the left or right main stem bronchus under video bronchoscopy (Fig. 1h; Supplementary Fig. 2l). The balloon was then expanded in the main stem bronchus to occlude airflow (Supplementary Fig. $2 \mathrm{~m}$ ) and enable single lung ventilation of the contralateral lung. Additionally, when obtaining measurements from the left lung the endotracheal tube was advanced slightly and the cuff was inflated to enable the occlusion of the right tracheal bronchus (i.e., pig bronchus). Once occluded, tidal volumes were reduced by half and pressure-volume loops and dynamic compliance of single lungs (injured or control) were acquired during single lung ventilation.

Bronchoalveolar lavage fluid analysis. BAL fluid samples were collected by wedging a $3.8 \mathrm{~mm}$ flexible bronchoscope (aScope 3, Ambu) into a subsegmental bronchus of an injured or control lung. Sterile normal saline $(5 \mathrm{~mL})$ was injected, aspirated, and collected in a sterile specimen trap (Busse Hospital Disposables). Gross images of BAL fluid samples were acquired throughout $36 \mathrm{~h}$ of crosscirculation using an interchangeable lens digital camera (Sony). The $\mathrm{pH}$ of BAL fluid samples was measured with a glass double junction microelectrode (Fisher Scientific). BAL fluid samples were centrifuged at $2164 \times g$ for $10 \mathrm{~min}$ at $4^{\circ} \mathrm{C}$. Snapfrozen supernatants were stored at $-80^{\circ} \mathrm{C}$ until further processing.

Cytopathology. The cellular contents of BAL fluid samples were visualized by Kwik-Diff staining or periodic acid-Schiff staining of BAL fluid smears and quantified using a previously described automatic cell counting algorithm (ImageJ) ${ }^{63}$ (Supplementary Fig. 4b).

Molecular pathology. Inflammatory markers (GM-CSF, IFN $\gamma$, IL-1 $\alpha$, IL-1 $\beta$, IL1ra, IL-2, IL-4, IL-6, IL-8, IL-10, IL-12, IL-18, M30, and TNFa) in BAL fluid samples collected from injured and control lungs were analyzed in triplicate using either the Eve Technologies Discovery Assay Pig Cytokine Array or commercially available enzyme-linked immunosorbant assays (ELISAs) (Supplementary Table 9). Total protein concentrations of BAL fluid samples were determined using a protein assay (Pierce Coomassie Bradford Protein Assay Kit, ThermoFisher Scientific) with bovine serum albumin as a standard. Pepsin concentrations in BAL fluid samples were measured using a commercially available porcine pepsin ELISA kit (LS Bio) according to the manufacturer's instructions.

Microbial cultures. BAL fluid samples collected from injured and control lungs throughout $36 \mathrm{~h}$ of interventional cross-circulation were submitted to a diagnostic laboratory (Antech Diagnostics; Lake Success, NY) for bacterial cultures. Microbial cultures of BAL fluid samples collected from injured lungs $(n=8)$ and control lungs $(n=8)$ were negative for pathologic growth after $72 \mathrm{~h}$ in all cases. Of note, all contents within the organ chamber were sterile, and proper sterile technique was stringently adhered to during all tissue sampling and lung manipulation. Between each use the bronchoscope was disinfected and sterilized according to established protocols to prevent bronchoscopy-associated infection ${ }^{64}$

Exosomes. BAL fluid samples collected from injured and control lungs throughout $36 \mathrm{~h}$ of interventional cross-circulation were submitted for analysis of exosome total concentration, concentration as a function of size, distribution of mean particle size (diameter), and imaging (Supplementary Movie 8) (NanoSight Tracking Analysis, System Biosciences).

Acetylated LDL uptake assay. To assess the function and viability of the vascular endothelium ${ }^{65}$, at the conclusion of each procedure, biopsies of the pulmonary arteries and veins were collected and placed in a 96-well plate (BD Falcon). Acetylated LDL, Alexa Fluor 594 conjugate (ThermoScientific L35353) was diluted 1:200 in $1 \mathrm{X}$ DMEM/F12K (50/50) cell culture media (Corning). Wells received $150 \mu \mathrm{L}$ of media containing acetylated LDL. Media alone $(150 \mu \mathrm{L})$ was added to control wells containing vascular biopsies from each source. The multi-well plate was then covered and incubated at $37^{\circ} \mathrm{C}$ with gentle shaking for $4 \mathrm{~h}$. Next, samples were washed five times with phosphate-buffered saline followed by fixation in cold phosphate-buffered $4 \%$ paraformaldehyde for $48 \mathrm{~h}$. Samples were then embedded 
in paraffin, sectioned (5- $\mu \mathrm{m}$ thickness), de-paraffinization, stained with DAPI, and examined using a fluorescent microscope (Olympus FSX100).

Carboxyfluorescein succinimidyl ester uptake assay. To assess the viability of the pulmonary endothelium, carboxyfluorescein succinimidyl ester (CFSE; Affymetrix, eBioscience) was reconstituted in dimethyl sulfoxide at a concentration of $1.06 \mathrm{M}$ and protected from light. At the conclusion of each procedure, CFSE was delivered via intravascular injection with a 20 Ga needle. Following $15 \mathrm{~min}$ incubation, samples were washed five times with phosphate-buffered saline, fixed in cold phosphate-buffered $4 \%$ paraformaldehyde for $48 \mathrm{~h}$, fixed, embedded, deparaffinized, and imaged using an Olympus FSX100 microscope.

BODIPY-surfactant uptake assay. To assess the functional uptake and viability of type II pneumocytes ${ }^{66}$, delivery of a fluorescent BODIPY-labeled surfactant protein $\mathrm{B}$ (BODIPY-SPB) was performed into the distal regions of injured lungs. A flexible video bronchoscope and Renegade microcatheter system (Boston Scientific) enabled targeted delivery. Lung samples were collected after 30 min using a surgical stapler (Medtronic). Samples were then dissected, rinsed (Dulbecco's phosphatebuffered saline), and imaged immediately with an Olympus FSX100 microscope. Further resolution was obtained by incubating injured lung tissue samples $(2 \times 2$ $\mathrm{mm}$ ) collected after $36 \mathrm{~h}$ of cross-circulation with $20 \mathrm{ng} \mathrm{mL}^{-1}$ BODIPY-SPB for $30 \mathrm{~min}$ in a normoxic incubator at $37^{\circ} \mathrm{C}$. Next, specimens underwent staining for 10 min with CellMask Deep Red plasma-membrane stain, followed by five washings for 1 min each (Dulbecco's phosphate-buffered saline). Images were acquired with a two-photon confocal laser scanning microscope (Leica TCS SP8). Visualization of the fluorescent signal in a punctate pattern (lamellar bodies) within type II pneumocytes indicated surfactant uptake.

Metabolic activity assay. To assess changes in lung metabolism, lung tissue samples from injured and control lungs were collected at 0 and $36 \mathrm{~h}$ of crosscirculation. Lung parenchyma ( $250 \mu \mathrm{L}$ tissue volume; $n=8)$ was dissected in standard sterile fashion. Fine mincing and gentle homogenization was performed prior to sample placement in a 96-well plate. Alamar Blue Assay ${ }^{67}$ (ThermoFisher) reagent was diluted 1:10 in DMEM cell culture media with 10\% fetal bovine serum. Next, $100 \mu \mathrm{L}$ Alamar Blue reagent was added to wells containing lung sample homogenates. In addition, Alamar Blue alone $(100 \mu \mathrm{L})$ was added to empty wells (negative controls). The multi-well plate was covered and incubated at $37^{\circ} \mathrm{C}$ with gentle shaking for $2 \mathrm{~h}$. Following incubation, well contents were transferred into new 96-well plates, and absorbance (optical density, OD) was measured at $570 \mathrm{~nm}$ and normalized to absorbance at $600 \mathrm{~nm}$. Following the metabolic activity assay, the DNA content of each sample was quantified using a Quanti-iT PicoGreen dsDNA Assay kit (Invitrogen) according to the manufacturer's instructions. Sample digestion was carried out in $250 \mu \mathrm{g}$ papain $\mathrm{mL}^{-1}$ at $60^{\circ} \mathrm{C}$ for $4 \mathrm{~h}$ and mixed with PicoGreen reagent. To quantify DNA using a standard curve, fluorescence emission was measured at $520 \mathrm{~nm}$ with excitation at $480 \mathrm{~nm}$.

Myeloperoxidase activity assay. To study the activity of myeloperoxidase (MPO), tissue samples were collected at multiple time points throughout crosscirculation. Lung tissue sections $(\geq 10 \mathrm{mg}$ ) were snap frozen in liquid nitrogen and stored at $-80^{\circ} \mathrm{C}$. MPO assay was conducted according to the manufacturer's instructions (Abcam, ab111749)

Thermography. Thermal images of the lungs were captured at selected time points throughout the cross-circulation procedure using a T430sc infrared camera (FLIR) and ResearchIR v. 4.40 .6 software (FLIR). Lung surface temperatures were averaged over isomorphic regions of interest in injured and control lungs and plotted relative to the average blood temperature during cross-circulation.

Hypothermic probe test. To standardize background reflectance and atmospheric effects ${ }^{68}$ prior to each hypothermic probe test, the lung was maintained for a minimum of $10 \mathrm{~min}$ enclosed within the organ chamber to allow lung surface temperatures to equilibrate to ambient conditions. For each hypothermic probe test, real-time calibrated thermal videos of the hypothermic probe test were recorded at 60 frames $\mathrm{s}^{-1}$. The organ chamber was opened, and a hypothermic probe (aluminum alloy tip, diameter: $8 \mathrm{~mm}$ ) maintained at $4{ }^{\circ} \mathrm{C}$ was immediately and consistently brought into physical contact with the pleural surface of injured or control lungs at pre-determined, designated locations for a duration of $1 \mathrm{~s}$. Contact pressures were calibrated and then standardized across all tests and time points using a manual push-pull force gauge (Topac). Thermal videography of lung regions topically cooled by thermophysical contact with the hypothermic probe was acquired for non-invasive quantitative assessment of localized rewarming in injured and control lungs (Supplementary Movie 7). Thermographic images were extracted from raw video sequences at $15 \mathrm{~s}$ intervals. Differences in surface temperatures between topically cooled regions and surrounding surface areas were quantified throughout $36 \mathrm{~h}$ of cross-circulation using video thermography and image analysis software (ImageJ $1.51 \mathrm{~m} 9$ ). Extracted images were subjected to image processing wherein average pixel values were measured in congruent regions of topically cooled areas and areas unaffected by hypothermic cooling. Differences in mean pixel values were calculated and plotted as a function of time.

Immunohistochemical staining. Following de-paraffinization, lung sections were subjected to boiling citrate buffer ( $\mathrm{pH}$ 6.0) for antigen retrieval, and blocked with $10 \%$ normal goat serum in phosphate-buffered saline for $2 \mathrm{~h}$ at room temperature. Next, primary antibodies were diluted 1:100, applied, and incubated for $12 \mathrm{~h}$ at $4{ }^{\circ} \mathrm{C}$ or $4 \mathrm{~h}$ at room temperature. Secondary antibodies were diluted 1:200 and incubated for $1 \mathrm{~h}$ at room temperature. Sections were mounted in Vectashield Mounting Medium with DAPI (Vector Laboratories), and coverslips were applied. Images were obtained using an Olympus FSX100 microscope. To detect late apoptosis, a terminal deoxynucleotidyl transferase-mediated deoxyuridine triphosphate nick end-labeling (TUNEL) assay was used. Immunohistochemical stains for aquaporin 5 (Abcam, ab78486), caspase 3 (Abcam, ab13847), CD163 (Abcam, ab87099), CD31 (Abcam, ab28364), connexin 43 (Abcam, ab11370), neutrophil elastase (Abcam, ab68672), surfactant protein C (Abcam, ab196677), zonula occludens-1 (Santa Cruz Biotechnology, sc-10804), and zonula occludens-3 (Abcam, ab205882) were conducted by the Herbert Irving Comprehensive Cancer Center molecular pathology services at Columbia University Medical Center. A list of antibodies and dilutions used is provided in Supplementary Table 10.

Histopathologic sample collection and analysis. The location of each lung wedge sample was randomized for each time point prior to the start of the experiment. Each lung was divided into 12 regions as shown in Supplementary Fig. 4a. To avoid sampling bias, a random number generator (www.random.org) was used to assign a lung region to each sample collection time point. Tissue sampling was restricted to the lower half of injured and control lungs to eliminate sampling bias in superior segments of upper lobes of injured lungs with better aeration. A surgical stapler with medium/thick reloads was used to obtain lung samples every $6 \mathrm{~h}$. Specimens were immediately fixed in cold phosphate-buffered $4 \%$ paraformaldehyde for $48-72 \mathrm{~h}$, embedded in paraffin, and sectioned at $3 \mu \mathrm{m}$ or $5 \mu \mathrm{m}$ thickness. All sections were stained for hematoxylin and eosin and examined under light microscopy. Additional sections were stained for Alcian blue ( $\mathrm{pH} 2)$, trichrome, silver reticulin, elastic van Gieson, and pentachrome by histology services in the Department of Molecular Pathology at Columbia University Medical Center.

Blinded pathologic review. Blinded pathologic review was performed by a pulmonary pathologist blinded to the study protocol. All slides (H\&E, immunohistochemical staining for neutrophil elastase and caspase 3, and TUNEL Supplementary Fig. 6 a, b; Supplementary Fig. 7f) were randomized, arbitrarily numbered, and delivered to the pathologist without reference to experimental time points or condition $5^{55}$. To evaluate recovery from injury resulting from aspiration of gastric contents, a modified lung injury score was developed from previously described methods ${ }^{56,57}$. Scoring (Supplementary Table 6) was based on number of airway polymorphonuclear cells per high-power field (hpf) $\left(\times 40 ; 0.2 \mathrm{~mm}^{2} ; 0 \Rightarrow 0\right.$, $1 \Rightarrow 1-25,2 \Rightarrow 26-50,3 \Rightarrow>50$ ), number of alveolar polymorphonuclear cells per hpf $\left(\times 40 ; 0.2 \mathrm{~mm}^{2} ; 0 \Rightarrow 0,1 \Rightarrow 1-25,2 \Rightarrow 26-50,3 \Rightarrow>50\right)$, alveolar edema $(0 \Rightarrow$ $<5 \%$ of all alveoli contained edema fluid, $1 \Rightarrow 6-25 \%, 2 \Rightarrow 26-50 \%, 3 \Rightarrow>50 \%$ ), interstitial infiltrate (number of lymphocytes and neutrophils in the interstitium around vessels and airways and in alveolar septa and pleura; $0 \Rightarrow 0$ per hpf, $1 \Rightarrow<50$ per hpf, $2 \Rightarrow 50-100$ per hpf, $3 \Rightarrow>100$ per hpf), interstitial edema (perivascular and peribronchial spaces expanded with edematous fluid; $0 \Rightarrow 0$ per hpf, $1 \Rightarrow 1 \mathrm{x}$ width vessel media, $2 \Rightarrow \geq 2 \mathrm{x}$ width vessel media), and total number of apoptotic cells per hpf $\left(\times 40 ; 0.2 \mathrm{~mm}^{2} ; 0 \Rightarrow<2,1 \Rightarrow 3-5,2 \Rightarrow 6-10,3 \Rightarrow>10\right)$. Numbers for early and late apoptosis, as quantified by caspase 3 and TUNEL, respectively, were combined into a single apoptosis score. Lung injury scores of injured and control lungs were compared at $6 \mathrm{~h}$ after gastric aspiration and periodically throughout interventional cross-circulation procedures as an index of lung injury following unilateral delivery of standardized gastric contents.

Scanning electron microscopy. Lung samples were obtained from multiple time points $(0,18$, and $36 \mathrm{~h}$ of cross-circulation), and fixed in formalin for $48 \mathrm{~h}$. Specimens were then rinsed in $70 \%$ ethanol, frozen, and lyophilized. Samples were imaged using Zeiss GeminiSEM 300 with accelerating voltage of $2.5 \mathrm{kV}$.

Transmission electron microscopy. Lung samples were obtained from multiple time points $(0,18$, and $36 \mathrm{~h}$ of cross-circulation), and fixed with $2.5 \%$ glutaraldehyde in $0.1 \mathrm{M}$ Sorensen's buffer ( $\mathrm{pH}$ 7.2). Next, lung samples were post-fixed with $1 \% \mathrm{OsO}_{4}$ in Sorenson's buffer for $1 \mathrm{~h}$, and following dehydration, tissues were embedded in Lx-112 (Ladd Research Industries). Sectioning was performed on a PT-XL ultramicrotome ( $60 \mathrm{~nm}$ thickness). Samples were then stained with uranyl acetate and lead citrate, and examined with a JEOL JEM-1200 EXII electron microscope. Images capture was performed using an ORCA-HR digital camera (Hamamatsu) and recorded with AMT Image Capture Engine v.602.569.

Statistical analysis. No data were excluded from analysis. One-way analysis of variance with Tukey's multiple comparison post hoc tests and Student's $t$-tests were 
performed using Prism Version 7.0e (GraphPad). A value of $p<0.05$ was considered statistically significant.

Study design. The study was designed as a proof-of-concept study $(n=8)$ for the purposes of testing interventional procedures and demonstrating the ability to support and recover the function of acutely injured lungs with cross-circulation for $36 \mathrm{~h}$, during which standard therapeutic interventions could be performed and the functional recovery of lungs assessed. Our hypothesis was that cross-circulation could provide a sufficiently robust level of extracorporeal support to enable multiple therapeutic interventions and recovery of extracorporeal lung function. The study was conducted with the minimum number of animals needed to demonstrate reproducibility and achieve statistical significance between time points. Data from this initial study will be used to conduct power analysis for subsequent investigations. Collection of all samples was performed as technical replicates in triplicate.

Randomization of sampling. Samples of extracorporeal lung collected for histologic, microscopic, and pathologic analyses were collected randomly during procedures according to a pre-determined lung map (Supplementary Fig. 4a) with 12 regions arbitrarily numbered for each lung.

Blinded review. All analytical assessments were blinded to the maximum practical extent. Pathologic analysis was performed by an independent expert to eliminate bias.

Reporting summary. Further information on research design is available in the Nature Research Reporting Summary linked to this article.

\section{Data availability}

The authors declare that all data reported in the paper and Supplementary Information will be made available to other investigators if requested. The source data underlying Fig. 2c-i, n; Fig. 3c-e, l; Fig. 4c; Fig. 5d; Fig. 6d-f,o are provided in the Source Data file.

Received: 5 September 2018 Accepted: 1 April 2019

Published online: 07 May 2019

\section{References}

1. Rana, A. et al. Survival benefit of solid-organ transplant in the United States. JAMA Surg. 150, 252-259 (2015).

2. Giwa, S. et al. The promise of organ and tissue preservation to transform medicine. Nat. Biotechnol. 35, 530-542 (2017).

3. Ware, L. B. et al. Assessment of lungs rejected for transplantation and implications for donor selection. Lancet 360, 619-620 (2002).

4. Wobma, H. \& Vunjak-Novakovic, G. Tissue engineering and regenerative medicine 2015: a year in review. Tissue Eng. Part B Rev. 22, 101-113 (2016).

5. Bittle, G. J. et al. The use of lung donors older than 55 years: a review of the United Network of Organ Sharing database. J. Heart Lung Transplant. 32, 760-768 (2013).

6. Petersen, T. H. et al. Tissue-engineered lungs for in vivo implantation. Science 329, 538-541 (2010).

7. Cypel, M. \& Keshavjee, S. Extending the donor pool: rehabilitation of poor organs. Thorac. Surg. Clin. 25, 27-33 (2015).

8. Cypel, M. et al. Normothermic ex vivo lung perfusion in clinical lung transplantation. N. Engl. J. Med. 364, 1431-1440 (2011).

9. Wallinder, A. et al. Transplantation of initially rejected donor lungs after ex vivo lung perfusion. J. Thorac. Cardiovasc. Surg. 144, 1222-1228 (2012).

10. Nakajima, D. et al. Ex vivo perfusion treatment of infection in human donor lungs. Am. J. Transplant. 16, 1229-1237 (2016).

11. Nakajima, D. et al. Lung lavage and surfactant replacement during ex vivo lung perfusion for treatment of gastric acid aspiration-induced donor lung injury. J. Heart Lung Transplant. 36, 577-585 (2017).

12. Inci, I. et al. Reconditioning of an injured lung graft with intrabronchial surfactant instillation in an ex vivo lung perfusion system followed by transplantation. J. Surg. Res. 184, 1143-1149 (2013).

13. Cypel, M. et al. Functional repair of human donor lungs by IL-10 gene therapy. Sci. Transl. Med. 1, 4ra9-4ra9 (2009).

14. Machuca, T. N. et al. Safety and efficacy of ex vivo donor lung adenoviral IL10 gene therapy in a large animal lung transplant survival model. Hum. gene Ther. 28, 757-765 (2017).

15. McAuley, D. F. et al. Clinical grade allogeneic human mesenchymal stem cells restore alveolar fluid clearance in human lungs rejected for transplantation. Am. J. Physiol. Lung Cell Mol. Physiol. 306, L809-L815 (2014).

16. Lee, J. W., Fang, X., Gupta, N., Serikov, V. \& Matthay, M. A. Allogeneic human mesenchymal stem cells for treatment of E. coli endotoxin-induced acute lung injury in the ex vivo perfused human lung. Proc. Natl Acad. Sci. USA 106, 16357-16362 (2009).
17. Kellar, C. Solid organ transplantation overview and delection criteria. Am. J. Manag. Care 21, S4-S11 (2015).

18. Marik, P. E. Aspiration pneumonitis and aspiration pneumonia. N. Engl. J. Med. 344, 665-671 (2001).

19. Engelhardt, T. \& Webster, N. Pulmonary aspiration of gastric contents in anaesthesia. Br. J. Anaesth. 83, 453-460 (1999).

20. Khalifé-Hocquemiller, T., Sage, E., Dorfmuller, P., Eddahibi, S. \& Fadel, E. 394 ex vivo perfusion worsened lung injuries induced by gastric acid aspiration in pigs. J. Heart Lung Transplant. 30, S135-S136 (2011).

21. Meers, C. M. et al. A model of ex vivo perfusion of porcine donor lungs injured by gastric aspiration: a step towards pretransplant reconditioning. J. Surg. Res. 170, e159-e167 (2011).

22. Inci, I. et al. Ex vivo reconditioning of marginal donor lungs injured by acid aspiration. J. Heart Lung Transplant. 27, 1229-1236 (2008).

23. Khalifé-Hocquemiller, T. et al. Exogenous surfactant attenuates lung injury from gastric-acid aspiration during ex vivo reconditioning in pigs. Transplantation 97, 413-418 (2014).

24. O'Neill, J. D. et al. Cross-circulation for extracorporeal support and recovery of the lung. Nat. Biomed. Eng. 1, 0037 (2017).

25. Fraisse, A. et al. Hemodynamics in experimental gastric juice induced aspiration pneumonitis. Intensive Care Med. 33, 300-307 (2007).

26. Reece, T. B. et al. Adenosine A $2 \mathrm{~A}$ receptor activation reduces inflammation and preserves pulmonary function in an in vivo model of lung transplantation. J. Thorac. Cardiovasc. Surg. 129, 1137-1143 (2005).

27. Mulloy, D. P. et al. Ex vivo rehabilitation of non-heart-beating donor lungs in preclinical porcine model: delayed perfusion results in superior lung function. J. Thorac. Cardiovasc. Surg. 144, 1208-1216 (2012).

28. Qazi, K. R. et al. Proinflammatory exosomes in bronchoalveolar lavage fluid of patients with sarcoidosis. Thorax 65, 1016-1024 (2010).

29. Oosthuyzen, W. et al. Quantification of human urinary exosomes by nanoparticle tracking analysis. J. Physiol. 591, 5833-5842 (2013).

30. Kim, J. et al. Controlled delivery and minimally invasive imaging of stem cells in the lung. Sci. Rep. 7, 13082 (2017).

31. Kim, J., O'Neill, J. D., Dorrello, N. V., Bacchetta, M. \& Vunjak-Novakovic, G. Targeted delivery of liquid microvolumes into the lung. Proc. Natl Acad. Sci. USA 112, 11530-11535 (2015).

32. Villagra, A. et al. Recruitment maneuvers during lung protective ventilation in acute respiratory distress syndrome. Am. J. Respir. Crit. care Med. 165, 165-170 (2002).

33. Fan, E. et al. Recruitment maneuvers for acute lung injury: a systematic review. Am. J. Respir. Crit. care Med. 178, 1156-1163 (2008).

34. Janda, M., Scheeren, T. W. \& Nöldge-Schomburg, G. F. Management of pulmonary aspiration. Best. Pract. Res. Clin. Anaesthesiol. 20, 409-427 (2006).

35. Abraham, V. et al. Heterocellular gap junctional communication between alveolar epithelial cells. Am. J. Physiol. Lung Cell Mol. Physiol. 280, L1085-L1093 (2001).

36. Meers, C. M. et al. A porcine model of acute lung injury by instillation of gastric fluid. J. Surg. Res. 166, e195-e204 (2011).

37. Kennedy, T. P. et al. Acute acid aspiration lung injury in the rat: biphasic pathogenesis. Anesth. Analg. 69, 87-92 (1989).

38. Hu, X., Lee, J. S., Pianosi, P. T. \& Ryu, J. H. Aspiration-related pulmonary syndromes. Chest 147, 815-823 (2015).

39. Nader, N. D., McQuiller, P. S., Raghavendran, K. \& Knight, P. R. The role of alveolar macrophages in the pathogenesis of aspiration pneumonitis. Immunol. Investig. 36, 457-471 (2007).

40. García, J. et al. Evaluation of inflammatory cytokine secretion by human alveolar macrophages. Mediat. Inflamm. 8, 43-51 (1999).

41. Knight, P. R., Druskovich, G., Tait, A. R. \& Johnson, K. J. The role of neutrophils, oxidants, and proteases in the pathogenesis of acid pulmonary injury. Anesthesiology 77, 772-778 (1992).

42. Nemzek, J. A. Mechanistic approaches to acid aspiration and pneumonia must look beyond tumor necrosis factor-a levels. Crit. Care Med. 33, 1877-1878 (2005).

43. Beck-Schimmer, B. \& Bonvini, J. M. Bronchoaspiration: incidence, consequences and management. Eur. J. Anaesthesiol. 28, 78-84 (2011).

44. Rodríguez-Moya, V. S. et al. Exogenous surfactant and alveolar recruitment in the treatment of the acute respiratory distress syndrome. Clin. Respir. J. 11, 1032-1039 (2017)

45. Eijking, E., Gommers, D., So, K., Vergeer, M. \& Lachmann, B. Surfactant treatment of respiratory failure induced by hydrochloric acid aspiration in rats. Anesthesiology 78, 1145-1151 (1993).

46. Tusman, G., Böhm, S., Vazquez de Anda, G., Do Campo, J. \& Lachmann, B. 'Alveolar recruitment strategy'improves arterial oxygenation during general anaesthesia. Br. J. Anaesth. 82, 8-13 (1999).

47. Leme, A. C. et al. Effect of intensive vs moderate alveolar recruitment strategies added to lung-protective ventilation on postoperative pulmonary complications: a randomized clinical trial. Jama 317, 1422-1432 (2017).

48. Frerichs, I., Dargaville, P. A., van Genderingen, H., Morel, D. R. \& Rimensberger, P. C. Lung volume recruitment after surfactant administration 
modifies spatial distribution of ventilation. Am. J. Respir. Crit. Care Med. 174, 772-779 (2006).

49. Metheny, N. A. et al. Tracheobronchial aspiration of gastric contents in critically ill tube-fed patients: frequency, outcomes, and risk factors. Crit. Care Med. 34, 1007 (2006).

50. Stovold, R. et al. Pepsin, a biomarker of gastric aspiration in lung allografts: a putative association with rejection. Am. J. Respir. Crit. Care Med. 175, 1298-1303 (2007).

51. Ward, C. et al. Pepsin like activity in bronchoalveolar lavage fluid is suggestive of gastric aspiration in lung allografts. Thorax 60, 872-874 (2005).

52. Hunt, E. B., Sullivan, A., Galvin, J., MacSharry, J. \& Murphy, D. M. Gastric aspiration and its role in airway inflammation. Open Respir. Med. J. 12, 1 (2018).

53. Modelska, K., Pittet, J.-F., Folkesson, H. G., Courtney Broaddus, V. \& Matthay, M. A. Acid-induced lung injury: protective effect of anti-interleukin8 pretreatment on alveolar epithelial barrier function in rabbits. Am. J. Respir. Crit. Care Med. 160, 1450-1456 (1999).

54. Folkesson, H., Matthay, M., Hebert, C. \& Broaddus, V. Acid aspirationinduced lung injury in rabbits is mediated by interleukin-8-dependent mechanisms. J. Clin. Investig. 96, 107 (1995).

55. van Westerloo, D. J. et al. Aspiration pneumonitis primes the host for an exaggerated inflammatory response during pneumonia. Crit. Care Med. 33, 1770-1778 (2005).

56. Rotta, A. T. et al. Gastric acid and particulate aspiration injury inhibits pulmonary bacterial clearance. Crit. Care Med. 32, 747-754 (2004).

57. Vassiliou, A. G. et al. Differential expression of aquaporins in experimental models of acute lung injury. In vivo 31, 885-894 (2017).

58. Whitsett, J. A. \& Weaver, T. E. Hydrophobic surfactant proteins in lung function and disease. N. Engl. J. Med. 347, 2141-2148 (2002).

59. Mikulska, D. Contemporary applications of infrared imaging in medical diagnostics. Ann. Acad. Med. Stetin. 52, 35-39 (2006).

60. Motoyama, H. et al. Novel thermographic detection of regional malperfusion caused by a thrombosis during ex vivo lung perfusion. Interact. Cardiovasc. Thorac. Surg. 20, 242-247 (2014).

61. Sachs, D. H. et al. TRANSPLANTATION IN MINIATURE SWINE: I. Fixation of the major histocompatibility complex. Transplantation 22, 559-567 (1976).

62. Judge, E. P. et al. Anatomy and bronchoscopy of the porcine lung. A model for translational respiratory medicine. Am. J. Respir. Cell Mol. Biol. 51, 334-343 (2014).

63. Papadopulos, F. et al. Common tasks in microscopic and ultrastructural image analysis using ImageJ. Ultrastruct. Pathol. 31, 401-407 (2007).

64. Mehta, A. C. et al. American College of Chest Physicians and American Association for Bronchology Consensus Statement: prevention of flexible bronchoscopy-associated infection. Chest 128, 1742-1755 (2005).

65. Voyta, J. C., Via, D. P., Butterfield, C. E. \& Zetter, B. R. Identification and isolation of endothelial cells based on their increased uptake of acetylated-low density lipoprotein. J. cell Biol. 99, 2034-2040 (1984).

66. Islam, M. N., Gusarova, G. A., Monma, E., Das, S. R. \& Bhattacharya, J. Factin scaffold stabilizes lamellar bodies during surfactant secretion. Am. J. Physiol. Lung Cell Mol. Physiol. 306, L50-L57 (2014).

67. Rampersad, S. N. Multiple applications of Alamar Blue as an indicator of metabolic function and cellular health in cell viability bioassays. Sensors 12 12347-12360 (2012)
68. Tattersall, G. J. Infrared thermography: a non-invasive window into thermal physiology. Comp. Biochem. Physiol. Part A Mol. Integr. Physiol. 202, 78-98 (2016).

\section{Acknowledgements}

The authors would like to thank A. Griesemer for discussions of the experimental design D. Sachs for providing research animals; the Institute of Comparative Medicine veterinary staff, including K. Fragoso and A. Rivas for their support of animal studies; L. Cohen-Gould for transmission electron microscopy imaging; the Herbert Irving Comprehensive Cancer Center Molecular Pathology Shared Resources, including T. Wu, D. Sun, and R. Chen for help with analytics; J. Bhattacharya for providing experimental reagents. YW. Chen for live cell imaging. BLES Biochemicals, Inc. provided the bovine lipid extract surfactant used in the study. The authors gratefully acknowledge funding support from the National Institutes of Health (grants HL134760, EB002520, and HL007854), the Richard Bartlett Foundation, and the Mikati Foundation.

\section{Author contributions}

B.A.G., J.D.O., J.K., G.V.-N, and M.B. designed the study. B.A.G., J.D.O., J.K., D.Q., S.C., K.F., M.Sim., R.D., M.S., K.C., S.P.H., H.M.W., A.E.H., A.R., and M.B. performed experiments. C.C.M. performed blinded pathologic assessment. B.A.G., J.D.O, G.V.-N, and M.B. co-analyzed data, co-wrote manuscript.

\section{Additional information}

Supplementary Information accompanies this paper at https://doi.org/10.1038/s41467019-09908-1.

Competing interests: The authors declare no competing interests.

Reprints and permission information is available online at http://npg.nature.com/ reprintsandpermissions/

Journal peer review information: Nature Communications thanks Dirk Van Raemdonck and the other anonymous reviewers for their contribution to the peer review of this work.

Publisher's note: Springer Nature remains neutral with regard to jurisdictional claims in published maps and institutional affiliations.

Open Access This article is licensed under a Creative Commons Attribution 4.0 International License, which permits use, sharing, adaptation, distribution and reproduction in any medium or format, as long as you give appropriate credit to the original author(s) and the source, provide a link to the Creative Commons license, and indicate if changes were made. The images or other third party material in this article are included in the article's Creative Commons license, unless indicated otherwise in a credit line to the material. If material is not included in the article's Creative Commons license and your intended use is not permitted by statutory regulation or exceeds the permitted use, you will need to obtain permission directly from the copyright holder. To view a copy of this license, visit http://creativecommons.org/ licenses/by/4.0/.

(C) The Author(s) 2019 